\title{
A Random Walk with Heavy Flavours
}

\author{
Surasree Mazumder, ${ }^{1}$ Trambak Bhattacharyya, ${ }^{1}$ and Santosh K. Das ${ }^{2}$ \\ ${ }^{1}$ Variable Energy Cyclotron Center, 1/AF, Bidhan Nagar, Kolkata 700064, India \\ ${ }^{2}$ Department of Physics and Astronomy, University of Catania, Via S. Sofia 64, 95125 Catania, Italy
}

Correspondence should be addressed to Surasree Mazumder; surasree.mazumder@gmail.com

Received 14 March 2013; Accepted 5 June 2013

Academic Editor: Jan E. Alam

Copyright (C) 2013 Surasree Mazumder et al. This is an open access article distributed under the Creative Commons Attribution License, which permits unrestricted use, distribution, and reproduction in any medium, provided the original work is properly cited.

We focus on evaluating transport coefficients like drag and diffusion of heavy quarks (HQ) passing through quark-gluon plasma using perturbative QCD (pQCD). Experimental-observable-like nuclear suppression factor $\left(R_{A A}\right)$ of HQ is evaluated for both zero and nonzero baryonic chemical potential $\left(\mu_{B}\right)$ scenarios using Fokker-Planck equation. Theoretical estimates of $R_{A A}$ are contrasted with experiments.

\section{Introduction}

When nuclear matter is subjected to an ambience of very high density, the individual quarks and gluons would no longer be confined within the hadrons but melt into a deconfined state of quarks and gluons. Just after the discovery of asymptotic freedom [1-3], Collins and Perry [4] also suggested that at very high density the degrees of freedom of the strongly interacting matters are not hadrons but quarks and gluons. The same is true when QCD vacuum is excited to high temperatures, too [5]. With increasing temperature, new and new hadrons are produced thereby increasing the corresponding number density, and at a certain temperature, there is an overlap of hadrons. Such a phase of matter is called quark-gluon plasma (QGP) and its study needs QCD, the theory of strong interaction which is extremely successful in vacuum, to be applied in a thermal medium. So, the deconfined state of quarks and gluons gives an opportunity to peruse "condensed matter physics" of elementary particles in the new domain of nonabelian gauge theory.

Lattice-QCD-based calculations predict that the typical value of the temperature for the quark-hadron transition, $T_{c}$, is $\sim 170 \mathrm{MeV}[6,7]$ (latest lattice QCD results show that $T_{c} \sim$ $160 \mathrm{MeV}$ [8-10]). According to the cosmological big bang model, the universe has undergone several phase transitions (GUT, electroweak, quark to hadron, etc.) at different stages of its evolution. The quark-hadron transition occurred when the universe was few microseconds ( $\mu$ s) old and this is the only transition which can be accessed in the laboratory currently. The study of quark-hadron transition demands special importance in understanding the evolution of the $\mu$ s old early universe. The issue is very crucial for astrophysics too, as the core of the compact astrophysical objects like neutron stars may contain quark matter at high baryon density and low temperature. So there is a multitude of reasons behind creating QGP in laboratories.

Temperature and energy density required to produce QGP in the laboratory can be achieved by colliding heavy ions at relativistic energies, under controlled laboratory environment. The nuclear collisions at Relativistic Heavy Ion Collider (RHIC) and the Large Hadron Collider (LHC) energies (200 GeV/A and $2.76 \mathrm{TeV} / \mathrm{A}$, resp.) are aimed at creating QGP. Once the QGP medium is created, we must try to understand the transport properties of the medium, that is, whether it is liquid or gas. The study of the transport coefficients of strongly correlated system is a field of high contemporary interest both theoretically and experimentally. In one hand, the calculation of the lower bound on the shear viscosity $(\eta)$ to entropy density $(s)$ ratio $(\eta / s)$ within the frame work of AdS/CFT model [11] has ignited enormous interests among the theorists. On the other hand, the experimental study of the $\eta / s$ for cold atomic systems and QGP and their similarities has generated huge interest across various branches of physics (see [12] for a review). 
In general, the interaction of probes with a medium brings out useful information about the nature of the medium. As the magnitude of the transport coefficients is sensitive to the coupling strength, so these quantities qualify as useful quantities to characterize a medium.

In context of probing QGP, the heavy quarks (HQs), mainly, charm and bottom quarks, play a vital role. The reasons are as follows.

(i) HQ mass is significantly larger than the typically attained temperatures and other nonperturbative scales $M \gg T_{c}, \Lambda_{\mathrm{QCD}}$ (intrinsic energy scale for the strong interaction); that is, the production of HQs is essentially constrained to the early, primordial stage of a heavy-ion collision and they do not dictate the bulk properties of the matter. Therefore, the heavy flavours are the witness to the entire space-time evolution of the system.

(ii) Their thermalization time scale is larger by a factor of $m / T$, where $m$ is the mass of heavy quarks and $T$ is the temperature, than that of the light quarks and gluons and hence heavy quarks can retain the interaction history very effectively.

From experimental point of view, however, the issue of HQ thermalization in QGP can be addressed by measuring the elliptic flow $\left(v_{2}\right)$ of leptons from the decays of HQs. Moreover, the observed transverse momentum suppression $\left(R_{A A}\right)$ of leptons originating from the decays of $D$ and $B$ mesons produced in nuclear collisions as compared to those produced in proton + proton (pp) collisions at the same colliding energy [13-15] offers us an opportunity to estimate the drag and diffusion coefficients of QGP. (It is now possible to detect directly $D$ mesons at LHC detectors like ALICE, see [16]). Hence, no wonder that in the recent past a large number of attempts have been made to study both heavy flavour suppression [13,14] and elliptic flow [17] within the framework of perturbative QCD (pQCD) [18-34].

\section{Motion of Heavy Quarks (HQs) in QGP}

In the introduction, we have already discussed that HQs act as effective probes to look into the properties of QGP. As HQs are much heavier than the particles constituting the QGP thermal bath, one expects that they will execute Brownian motion in QGP medium $[35,36]$. The system under study, then, would have two components: (i) the QGP formed at an initial temperature $T_{i}$ and initial thermalization time $\tau_{i}$ consisting of light quarks and gluons and (ii) heavy quark, the Brownian particle formed due to hard collisions at very early stage of heavy ion collision. The momentum distribution of $\mathrm{HQ}$ is governed by a nonlinear integrodifferential equation which is the Boltzman transport equation (BTE) as

$$
\left[\frac{\partial}{\partial t}+\frac{\mathbf{p}}{E} \cdot \frac{\partial}{\partial \mathbf{x}}+\mathbf{F} \cdot \frac{\partial}{\partial \mathbf{p}}\right] f(x, \mathbf{p}, t)=\left[\frac{\partial f}{\partial t}\right]_{\text {collisions }} .
$$

F is the force exerted on the HQ by the surrounding colour field. $\mathbf{p}$ and $E$ denote the three momentums and the energy of the HQ, respectively. The right hand side of (1), which is called the collision integral, $C[f]$, is attributed to the QCD interactions of HQ with light quarks $(q)$, antiquarks $(\bar{q})$, and gluons (g). One should, in principle, solve this differential equation under the influence of potential involving interaction of HQs with light quarks/anti-quarks and the background colour field in the force term. But, here, we will set $\mathbf{F}=0$ and will treat QGP to be uniform. Therefore, the second and the third term of the left hand side of (1) vanish under these approximations. Again defining

$$
f(\mathbf{p}, t)=\frac{1}{V} \int d^{3} \mathbf{x} f(\mathbf{x}, \mathbf{p}, t)
$$

which is the normalized probability distribution in the momentum space, we have

$$
\frac{\partial f(\mathbf{p}, t)}{\partial t}=\left[\frac{\partial f}{\partial t}\right]_{\text {collisions }} .
$$

Equation (3) signifies that all variation of the distribution function of HQ with time is due to the collisions only.

\section{Formalism}

Our main aim is to determine the collision integral of the transport (3). Once, we determine certain form of $C[f]$, we can proceed towards solving the differential equation. There are lots of approximations through which the integrodifferential equation can be solved. Of course, under certain conditions, (3) can be reduced to the simple form

$$
\frac{\partial f(\mathbf{p}, t)}{\partial t}=-\frac{f-f_{0}}{\tau}
$$

which is a useful first approximation. Here, $f_{0}$ is the equilibrium distribution function and $\tau$ is the relaxation time that determines the rate at which the fluctuations in the system drive it to a state of equilibrium again. In this form, the equation is very easy to solve. But, our case is not so simple. We will deal with a more sophisticated approximation that leads to the Fokker-Planck equation [37].

To start with, we apply the Landau approximation which allows only soft scattering in the collision integral. If we define $w(\mathbf{p}, \mathbf{k})$ to be the rate of collisions which change the momentum of the HQ from $\mathbf{p}$ to $\mathbf{p}-\mathbf{k}$, we have

$$
\begin{aligned}
& {\left[\frac{\partial f}{\partial t}\right]_{\text {collisions }}} \\
& \quad=\int d^{3} \mathbf{k}[w(\mathbf{p}+\mathbf{k}, \mathbf{k}) f(\mathbf{p}+\mathbf{k})-w(\mathbf{p}, \mathbf{k}) f(\mathbf{p})] .
\end{aligned}
$$

The second part of the integral corresponds to all those transitions that remove HQ from momentum $\mathbf{p}$ to $\mathbf{p}-\mathbf{k}$ and therefore represents a net loss to the distribution function. Likewise, the first part of the integral represents a net gain to the distribution function of HQ. With these, (3) becomes

$$
\frac{\partial f(\mathbf{p}, t)}{\partial t}=\int d^{3} \mathbf{k}[w(\mathbf{p}+\mathbf{k}, \mathbf{k}) f(\mathbf{p}+\mathbf{k})-w(\mathbf{p}, \mathbf{k}) f(\mathbf{p})]
$$


Equation (6) is a linear equation in $f$. We can simplify it by assuming the previously discussed Landau approximation. Mathematically, this approximation amounts to assuming $w(\mathbf{p}, \mathbf{k})$ to fall off rapidly to zero with $|\mathbf{k}|$; that is, transition probability function, $w(\mathbf{p}, \mathbf{k})$, is sharply peaked around $|\mathbf{k}|=$ 0 . Therefore, if we expand the integrand in the right hand side of (6) in powers of $\mathbf{k}$, we have

$$
\begin{aligned}
w(\mathbf{p}+\mathbf{k}, \mathbf{k}) f(\mathbf{p}+\mathbf{k}) \approx & w(\mathbf{p}, \mathbf{k}) f(\mathbf{p})+\mathbf{k} \cdot \frac{\partial}{\partial \mathbf{p}}(w f) \\
& +\frac{1}{2} k_{i} k_{j} \frac{\partial^{2}}{\partial p_{i} \partial p_{j}}(w f) .
\end{aligned}
$$

Retaining terms up to the second order only, we obtain Fokker-Planck equation [37]

$$
\frac{\partial f}{\partial t}=\frac{\partial}{\partial p_{i}}\left[A_{i}(\mathbf{p}) f+\frac{\partial}{\partial p_{j}}\left[B_{i j}(\mathbf{p}) f\right]\right],
$$

where the kernels are defined as follows:

$$
\begin{gathered}
A_{i}=\int d^{3} \mathbf{k} w(\mathbf{p}, \mathbf{k}) k_{i}, \\
B_{i j}=\frac{1}{2} \int d^{3} \mathbf{k} w(\mathbf{p}, \mathbf{k}) k_{i} k_{j} .
\end{gathered}
$$

In the present formalism, we considered the elastic scattering of the HQ with the gluon, light quark, and the corresponding anti-quarks. All these processes contribute to determine $w(\mathbf{p}, \mathbf{k})[37]$ and, in turn, the above defined kernels. Now, to explore the physical significance of the $A$ and $B$ coefficients, let us consider $A_{i}=p_{i} \gamma(p)$ and $B_{i j}=D(p) \delta_{i j}$, which assume very low $\mathbf{p}$; that is, the medium of QGP is isotropic to the HQ. If, in this limit, we neglect all the derivatives of $A$ and $B$ coefficients with momentum of HQ, (8) reduces to

$$
\frac{\partial f}{\partial t}=\gamma \frac{\partial}{\partial \mathbf{p}} \cdot(\mathbf{p} f)+D\left[\frac{\partial}{\partial \mathbf{p}}\right]^{2} f .
$$

The method of solution of this equation is elaborately discussed in [37]. Now, if we want to extend this formalism to the regime where the momentum of the HQ is no longer small, that is, the particular kinematic domain where HQ becomes relativistic, obviously, we would like to know how the transport coefficients drag $(\gamma)$ and diffusion $(D)$ behave at high $\mathbf{p}$ region. In order to do so, we extrapolate the concept of isotropy to the higher momentum of HQ in such a way that only the first derivatives of the drag and diffusion coefficients are considered and the momentum dependence of $A$ and $B$ coefficients is encoded inside $\gamma$ and $D$. Therefore, the Fokker-Planck equation, under this approximation, in Cartesian coordinate system becomes [28]

$$
\begin{aligned}
\frac{\partial f}{\partial t}= & C_{1}\left(p_{x}, p_{y}, t\right) \frac{\partial^{2} f}{\partial p_{x}^{2}}+C_{2}\left(p_{x}, p_{y}, t\right) \frac{\partial^{2} f}{\partial p_{y}^{2}} \\
& +C_{3}\left(p_{x}, p_{y}, t\right) \frac{\partial f}{\partial p_{x}}+C_{4}\left(p_{x}, p_{y}, t\right) \frac{\partial f}{\partial p_{y}} \\
& +C_{5}\left(p_{x}, p_{y}, t\right) f+C_{6}\left(p_{x}, p_{y}, t\right),
\end{aligned}
$$

where,

$$
\begin{gathered}
C_{1}=D \\
C_{2}=D \\
C_{3}=\gamma p_{x}+2 \frac{\partial D}{\partial p_{T}} \frac{p_{x}}{p_{T}}, \\
C_{4}=\gamma p_{y}+2 \frac{\partial D}{\partial p_{T}} \frac{p_{y}}{p_{T}}, \\
C_{5}=2 \gamma+\frac{\partial \gamma}{\partial p_{T}} \frac{p_{x}^{2}}{p_{T}}+\frac{\partial \gamma}{\partial p_{T}} \frac{p_{y}^{2}}{p_{T}}, \\
C_{6}=0
\end{gathered}
$$

where the momentum $\mathbf{p}=\left(\mathbf{p}_{T}, p_{z}\right)=\left(p_{x}, p_{y}, p_{z}\right)$. We numerically solve (11) [38] with the boundary conditions $f\left(p_{x}, p_{y}, t\right) \rightarrow 0$ for $p_{x}, p_{y} \rightarrow \infty$, and the initial (at time $t=\tau_{i}$ ) momentum distribution of charm and bottom quarks is taken from MNR code [39]. It is evident from (11) that with the momentum-dependent transport coefficients the FP equation becomes complicated.

It is possible to write down the solution of the FP equation in closed analytical form [40] in the special case of momentum-independent drag and diffusion coefficients. To find out the solution $f$ for momentum independent drag and diffusion coefficients, we can consider the one-dimensional version of (10) as

$$
\frac{\partial f}{\partial t}=\gamma \frac{\partial}{\partial}(p f)+D \frac{\partial^{2} f}{\partial p^{2}},
$$

which is also called the Rayleigh's equation. For the initial condition $f(p, t=0)=\delta\left(p-p_{0}\right)$, the solution of $f$ is

$$
\begin{aligned}
f(p, t)= & \left(\frac{\gamma}{2 \pi D}\left(1-e^{-2 \gamma t}\right)\right)^{-1 / 2} \\
& \times \exp \left(-\frac{\gamma}{2 D} \frac{\left(p-p_{0} e^{-\gamma t}\right)^{2}}{1-e^{-2 \gamma t}}\right) .
\end{aligned}
$$

However, (14) is solution for a very simplified scenario and we will study the momentum dependence of drag and diffusion coefficients and their effects on $R_{A A}$ in the sections to come.

\section{Drag Coefficients}

4.1. Elastic Processes. We need to evaluate the drag coefficient as a function of temperature and momentum of HQ. The expression to evaluate collisional drag can be written as [37]

$$
\begin{aligned}
\gamma_{\text {coll }}= & \frac{1}{2 E_{p}} \int \frac{d^{3} \mathbf{q}}{(2 \pi)^{3} 2 E_{q}} \int \frac{d^{3} \mathbf{q}^{\prime}}{(2 \pi)^{3} 2 E_{q^{\prime}}} \\
& \times \int \frac{d^{3} \mathbf{p}^{\prime}}{(2 \pi)^{3} 2 E_{p^{\prime}}} \frac{1}{\gamma_{\mathrm{Q}}} \sum|M|^{2} \\
& \times(2 \pi)^{4} \delta^{4}\left(p+q-p^{\prime}-q^{\prime}\right) f^{\prime}(\mathbf{q})\left[1-\frac{\mathbf{p} \cdot \mathbf{p}^{\prime}}{p^{2}}\right],
\end{aligned}
$$




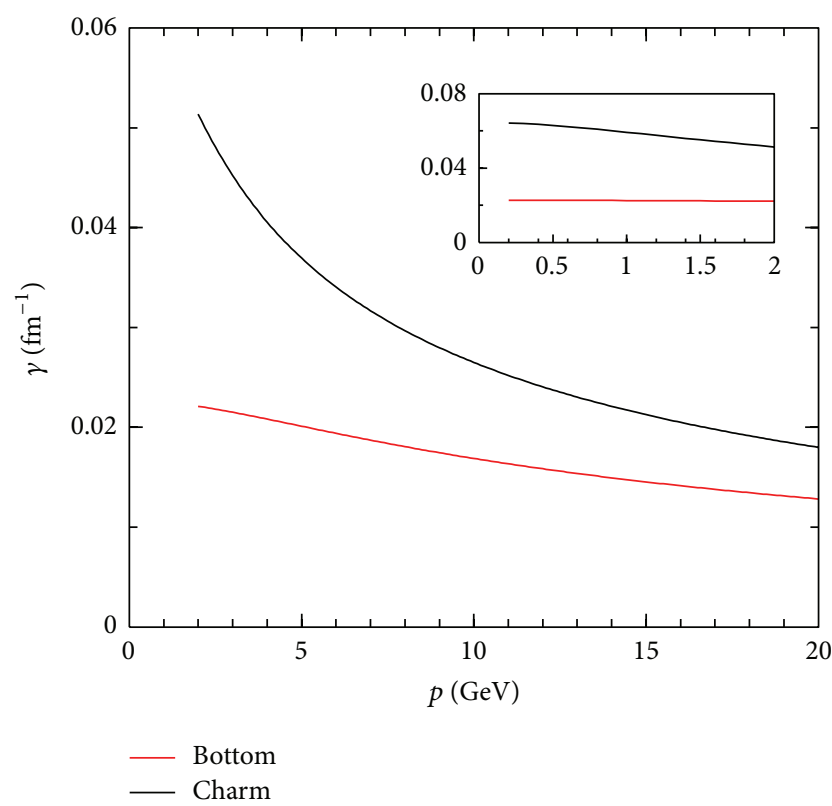

FIGURE 1: Drag coefficients of charm and bottom with their momentum at $T=300 \mathrm{MeV}$ assuming running strong coupling, $\alpha_{s}(T)$, and temperature-dependent Debye screening mass, $m_{D}(T)$ for gluon, quark, and antiquark scattering.

where $\mathbf{p}^{\prime}=\mathbf{p}-\mathbf{k}$ and $\mathbf{q}^{\prime}=\mathbf{q}+\mathbf{k}$. The scattering matrix elements are given explicitly in [41] where the $t$ channel divergence occurring due to very soft gluon exchange has been shielded by replacing $t$ in the denominator of matrix elements by $t-m_{D}^{2}$ in an ad hoc manner, where $t$ is the Mandelstam variable and $m_{D}$ is the thermal mass of gluon. However, the same problem can be approached from hard thermal loop (HTL) perturbation theory. The gluon propagator for $t$ channel diagram is then replaced by HTL propagator. But calculation of even the elastic matrix elements for HQs scattering with light quarks and gluons in this approach is very lengthy and radiative matrix elements are even clumsier. However, an outline of calculating collisional drag and diffusion coefficients in HTL perturbation theory approach is given for the interested readers in the Appendix. Here we proceed with the conventional process of shielding $t$ channel divergence with the gluon thermal mass, $m_{D}$.

The integrations in (15) have been performed using the standard techniques $[23-25,37]$. Results of the present calculation of drag coefficients are plotted with respect to momenta of charm and bottom (Figure 1). We can observe that the momentum dependence of $\gamma$ is nonnegligible for the shown momentum range. The value of $\gamma$ for charm due to collisional processes at $p=5 \mathrm{GeV}$ is about $0.036 \mathrm{fm}^{-1}$ which reduces to a value of $0.018 \mathrm{fm}^{-1}$ at $p=10 \mathrm{GeV}$ (can be compared with, e.g., [42-45]). In the inset, the drag coefficients of HQs due to elastic collision are plotted in the lower momentum region, where the drag remains more or less constant with $\mathbf{p}$. Therefore, it is clear that had we taken the value of drag at low momentum and extrapolated that value to higher momentum, the final result would have been overestimated. The diffusion coefficient of HQ can be evaluated from Einstein relation $D=\gamma M T$. Later, it will be seen

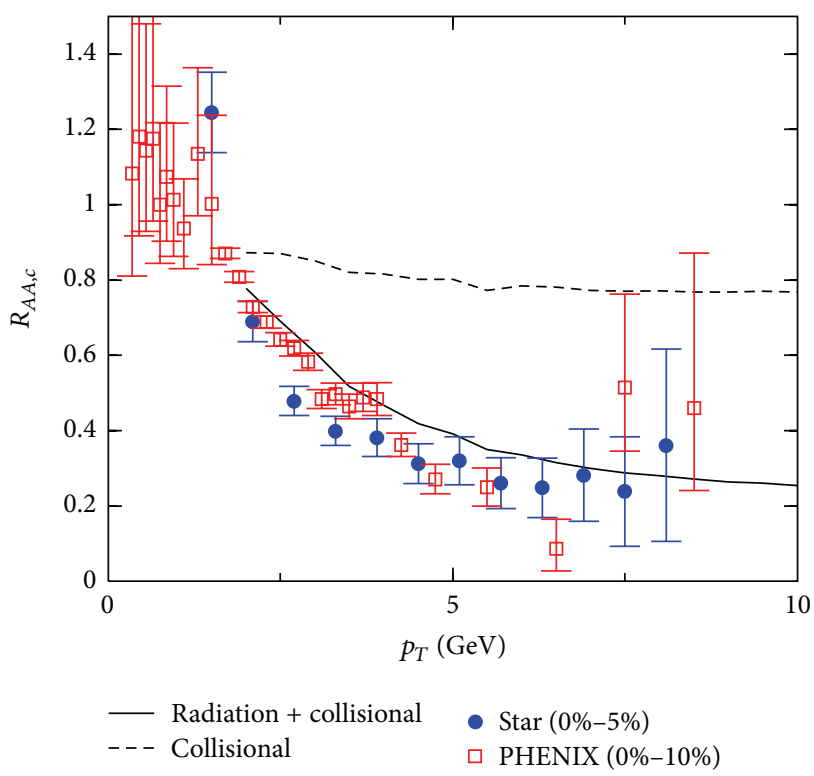

FIGURE 2: Suppression of transverse momentum of charm quarks in QGP as a function $p_{T}$.

that the momentum dependence of transport coefficients of a high energy HQ will have considerable effects on the nuclear suppression factor, $R_{A A}$ of $\mathrm{HQ}$.

However, there are also radiative processes taking place in the medium. The radiative drag can be obtained in the present formalism by computing the radiative energy loss of a HQ passing through QGP. In the next section, we will discuss the methods and intricacies of calculating the transport coefficients in radiative domain.

4.2. Radiative Processes. It is already known that the two mechanisms of energy loss of heavy quarks are collisional and radiative energy loss. Though at low transverse momentum $\left(p_{T}\right)$ region the collisional and radiative losses of HQs are comparable (see Figure 5), the radiative one tends to dominate with increasing momentum. So it is worthwhile, after discussing about evaluation of collisional transport coefficients (drag, diffusion) within the ambit of $T=0$ perturbative QCD (pQCD) [37] in the previous section, to contemplate on radiative processes and to inspect how $\mathrm{pQCD}$ approach can be utilized in finding out radiative transport coefficients.

It is well known from QED that for high energies radiative energy loss becomes dominant [46]. Also the hard thermal loop calculations in context of QCD show that the radiative energy loss contributes to the same order of strong coupling as that of collisional loss [47]. The above discussions tempt one to infer that the observed large suppression of heavy quarks at RHIC is predominantly due to bremsstrahlung processes $[25,28]$, at least at high $p_{T}$, but, as already said, the comparable values of energy losses in low momentum region leave an ample room for ambiguity in this statement particularly at "not-so-high" $p_{T}(\sim 2 \mathrm{GeV})$ region.

Theoretical estimate of nuclear suppression factor $\left(R_{A A}\right)$ for charm quarks in [28] shows $\sim 4$ times more suppression due to inclusion of radiation (Figure 2) with the same initial conditions. Inclusion of radiative processes leads to a good 
description of charm $R_{A A}$ at RHIC energies. At LHC energy $(2.76 \mathrm{TeV} / \mathrm{A})$, the collisional energy loss due to hard (momentum transfer $>2 \mathrm{GeV}$ ) collisions could be about one-third of the total [29]. The rest may be attributed to radiative loss.

From the discussion on collisional transport coefficients we know that (collisional) drag is given by [37]

$$
\gamma_{\text {coll }}=-\frac{1}{p}\left(\frac{d E}{d x}\right)_{\text {coll }},
$$

where $p$ is the momentum of the probe. We employ a similar argument and relate radiative energy loss $\left((d E / d x)_{\text {rad }}\right)$ to inelastic drag $\left(\gamma_{\mathrm{rad}}\right)$ in the same way. The effective drag obtained is a summation of collisional and radiative parts. As energy loss is related with the transport properties like drag offered by the medium, we must concentrate on more and more accurate determination of radiative energy loss which will enable us to understand the properties of QGP.

The radiative energy loss in general has been studied in [48-53] incorporating Landau-Pomeranchuk-Migdal (LPM) effect $[54,55]$, to be discussed later, due to multiple scattering. The authors of [56] treat the problem of radiative energy loss of HQs by building a model in scalar QCD approach. Attempts have been taken $[25,28,30]$ to incorporate the formalism of [48], the Gyulassy-Wang potential model (GWPM), to compute the radiative energy loss of heavy quarks traveling through QGP. In stead of going to the energy loss in GWPM approach directly, we may try to acquire some familiarity with the model and the notations used for the description of it.

\subsubsection{Gyulassy Wang Potential Model (GWPM) and Radiation} Spectrum. To analyze the multiple scattering and the induced gluon radiation in GWPM, certain simplifications have to be made. For example, [49] assumes static interaction between propagating parton and bath particles. This interaction is modelled by a static Debye screening potential. Now, it is possible to approximate the effective average random colour field produced by the bath particles by a potential provided the distance between two successive scatterers is large compared to colour screening length $\left(\mu^{-1}\right)$. The screened potential is given by

$$
\begin{aligned}
V_{A A^{\prime}}^{a}(\vec{q}) & =A_{A A^{\prime}}^{a}(\vec{q}) e^{-i \vec{q} \cdot \vec{x}} \\
& =g T_{A A^{\prime}}^{a} e^{-i \vec{q} \cdot \vec{x}} \\
\vec{q}^{2}+\mu^{2} &
\end{aligned}
$$

where $\mu$ is the colour screening mass, $T^{a}$ are the generators corresponding to the representation of target partons at $\vec{x}$ transferring (three) momentum $\vec{q}$, and $g$ is the coupling. The Feynman diagrams contributing to induced gluon radiation from a single quark-quark scattering are given in Figure 3.

The calculation of Feynman diagrams is done in lightcone coordinates $[57,58]$ where light-cone representations of four-vectors are done in the following way:

$$
\begin{aligned}
p & =\left(p^{+}, p^{-}, p_{\perp}\right) \\
& =\left(p_{0}+p_{3}, p_{0}-p_{3}, p_{\perp}\right) .
\end{aligned}
$$

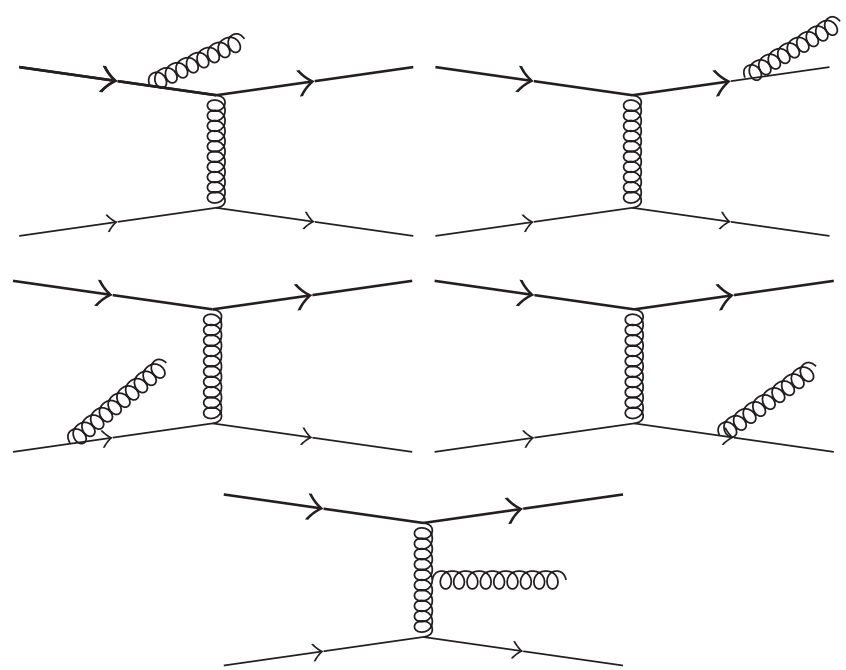

FIGURE 3: Feynman diagrams for gluon radiation from a single quark-quark $\left(q q^{\prime}\right)$ scattering.

In the potential model, one can neglect radiation from the target lines (external lines appearing at the bottom of gluon propagator in Figure 3) provided one decides to work in light cone gauge, $A^{+}=0$, for emitted gluon fields (For details see [59]). For radiation in the midrapidity region, the celebrated Gunion-Bertsch distribution formula (GB formula) for soft gluon radiation [60] can be reproduced from this approach.

We now discuss the GB formula and recent attempts to generalize. (Interested readers are referred to the Appendix for other such very recent endeavors.) We will see that this formula and/or its generalizations will play a significant role in finding out the energy loss due to gluon emission of quarks. The early attempts of generalizing GB formula [6163 ] consider the general gg $\rightarrow$ ggg matrix element elegantly written by [64] and factoring out the elastic gg $\rightarrow$ gg scattering amplitude to obtain the distribution of emitted gluon $\left(\sim\left|M_{\mathrm{gg} \rightarrow \mathrm{ggg}}\right|^{2} /\left|M_{\mathrm{gg} \rightarrow \mathrm{gg}}\right|^{2}\right)$. We can write the following form of gluon distribution [63], (for details, see Appendix of [63])

$$
\begin{aligned}
\frac{d n_{g}}{d^{2} k_{\perp} d \eta}=\left[\frac{d n_{g}}{d^{2} k_{\perp} d \eta}\right]_{\mathrm{GB}} & {\left[\left(1+\frac{t}{2 s}+\frac{5 t^{2}}{2 s^{2}}-\frac{t^{3}}{s^{3}}\right)\right.} \\
& -\left(\frac{3}{2 \sqrt{s}}+\frac{4 t}{s \sqrt{s}}-\frac{3 t^{2}}{2 s^{2} \sqrt{s}}\right) k_{\perp} \\
& \left.+\left(\frac{5}{2 s}+\frac{t}{2 s^{2}}+\frac{5 t^{2}}{s^{3}}\right) k_{\perp}^{2}\right],
\end{aligned}
$$

where $\eta$ is the rapidity of the radiated gluon, and the subscript GB has been used to indicate the gluon spectrum obtained using the approximation considered in [60] (see also [65]) which is generally given by

$$
\left[\frac{d n_{g}}{d^{2} k_{\perp} d \eta}\right]_{\mathrm{GB}}=\frac{C_{A} \alpha_{s}}{\pi^{2}} \frac{q_{\perp}^{2}}{k_{\perp}^{2}\left[\left(\vec{k}_{\perp}-\vec{q}_{\perp}\right)^{2}+m_{D}^{2}\right]},
$$


where $m_{D}=\sqrt{(2 \pi / 3) \alpha_{s}(T)\left(C_{A}+N_{F} / 2\right)} T$ is the thermal mass of the gluon $[66,67], N_{F}$ is the number of flavours contributing in the gluon self-energy loop, $C_{A}=3$ is the Casimir invariant for the $\mathrm{SU}(3)$ adjoint representation, $\alpha_{s}$ is the temperature-dependent strong coupling [68], $k_{\perp}$ is the transverse momentum of the emitted gluon, and $q_{\perp}$ is the transverse momentum transfer. In general, one introduces the thermal mass in the denominator of (20) to shield the divergence arising from collinearity (i.e., when $\vec{k}_{\perp}=\vec{q}_{\perp}$ ) of emitted gluon. But, for the present case (19) is written under the assumption that $\vec{q}_{\perp} \gg \vec{k}_{\perp}$, and hence there is no need to write the $m_{D}^{2}$ factor in GB spectrum.

Now, we must consider multiple scattering encountered by incoming particles, too. The many-body effect due to presence of medium results in interference of scattering amplitudes. This interference effect is called LPM effect [54, 55]. LPM effect is discussed in QED domain in [69]. LPM suppression can be understood in a qualitative manner as an interplay between two time scales, the formation time $\left(\tau_{f}\right)$ and the scattering time $\left(\tau_{\mathrm{sc}}\right), \tau_{F}$ is the time needed for the emission of the induced gluon. Actually, $\tau_{f}$ determines the time span after which a radiation can be separately identified from the parent parton from which the radiation is being given off. Now, a collision just before formation of the gluon results in suppression of the radiation. This destructive interference is called LPM effect and in the next section we will see that it puts a constraint on the phase space of the emitted gluon. If $k_{0}$ is the energy of the emitted (soft) gluon and $k_{\perp}$ is its transverse momentum, then formation time $\tau_{f} \sim$ $2 k_{0} / k_{\perp}^{2}$ [49]. When this $\tau_{f}$ is much less than collision time, that is, separation between two scattering centres, $L$, then the intensity of radiation is additive and Bethe-Heitler $(B H)$ limit is reached. In the factorization limit, $L \ll \tau_{f}$, the interference in radiation amplitude takes place and LPM effect dominates.

After this brief discussion on possible effects of multiple scattering, emitted gluon distribution due to multiple scattering in terms of that due to single scattering can be written as [49]

$$
\frac{d n_{g}^{(m)}}{d^{2} k_{\perp} d \eta}=C_{m}(k) \frac{d n_{g}^{(1)}}{d^{2} k_{\perp} d \eta},
$$

where " $m$ " stands for multiple scattering and " 1 " stands for single scattering. $C_{m}$ is called radiation formation factor characterizing the interference pattern due to multiple scattering. Naturally, in the $\mathrm{BH}$ limit, $C_{m} \approx m$, which implies that scattering amplitudes are just additive and the resultant intensity shows no interference pattern. On the other hand, the factorization limit gives $[49,50]$

$$
\begin{aligned}
C_{m}(k) \approx & \frac{8}{9}\left[1-\left(-\frac{1}{8}\right)^{m}\right] \quad \text { for quarks } \\
& \approx 2\left(1-\frac{1}{2^{m}}\right) \quad \text { for gluons. }
\end{aligned}
$$

Equation (22) shows that the interference effect due to many multiple scatterings for quarks leaves corresponding radiation spectrum a factor of $8 / 9$ of that due to single scattering.
It can also be checked that the gluon intensity radiated by gluon jet is $9 / 4$ times higher than that radiated by quark jets in multiple scattering. Thus, the LPM effect in QCD depends on colour representation due to nonabelian nature of the problem under discussion.

4.2.2. Energy Loss of Light Particles in GWPM. The energy loss in potential model can be evaluated by integrating over the transverse momentum and rapidity of emitted gluon. The phase space is, of course, constrained by LPM effect due to multiple scattering. The multiple scattering is implemented through the differential change of the factor $C_{m}$ with number of scattering $m, d C_{m} / d m$, which can be approximated as a $\theta$-function [49]. Also, the emitted gluon must have energy less than that of the parent parton. So the energy loss formula implementing the energy constraint gives

$$
\begin{aligned}
\Delta E_{\mathrm{rad}}= & \frac{E_{m+1}-E_{m}}{m} \\
& =\int d^{2} k_{\perp} d \eta \frac{d n_{g}}{d^{2} k_{\perp} d \eta} k_{0} \frac{C_{m+1}-C_{m}}{m} \theta\left(E-k_{\perp} \cosh \eta\right) \\
& =\int d^{2} k_{\perp} d \eta \frac{d n_{g}}{d^{2} k_{\perp} d \eta} k_{0} \frac{d C_{m}}{d m} \theta\left(E-k_{\perp} \cosh \eta\right) \\
& \sim \int d^{2} k_{\perp} d \eta \frac{d n_{g}}{d^{2} k_{\perp} d \eta} k_{0} \theta\left(\tau_{\mathrm{sc}}-\tau_{F}\right) \theta\left(E-k_{\perp} \cosh \eta\right),
\end{aligned}
$$

where $E$ is the energy of the parent parton and $k_{0}=k_{\perp} \cosh \eta$, $E_{m+1}$ denotes energy loss in $(m+1)$ th, collision and $E_{m}$ is the corresponding value in the $m$ th collision. The first $\theta$ function involving scattering time $\tau_{\text {sc }}$ [70] gives a lower limit of $k_{\perp}$, that is, $k_{\perp}>\Lambda \cosh \eta$. The second $\theta$-function yields $k_{\perp}<E / \cosh \eta$. Utilizing (23), [63] compares the energy loss (Figure 4) of a gluon jet passing through gluonic plasma obtained by generalized GB formulae given in [61-63]. We will see how (23) can be employed to find out HQ energy loss in the next section.

4.2.3. Energy Loss of HQs and Radiative Drag. While calculating the radiative energy loss of HQs [56] takes the GB distribution for small and moderate $x$ and for mass $m \neq 0$. The distribution, so obtained, can be shown to yield GB distribution when $x \ll 1$ and when $m=0$. However the authors of $[25,28]$ use the original GB distribution formula weighted by a radiative suppression factor, called "dead-cone" factor, originating due to mass of HQs. Since dead-cone factor plays an important role in radiative energy loss of heavy quarks, we will pause here to spend some words on dead-cone effect in QCD [71] and its generalizations.

The dead-cone suppression obtained in [71] actually has an analogy with radiated power distribution of a nonrelativistic, accelerating charge particle. The average power radiated per unit solid angle is given by [72]

$$
\frac{d P}{d \Omega} \propto|\dot{\vec{\beta}}|^{2} \sin ^{2} \theta
$$




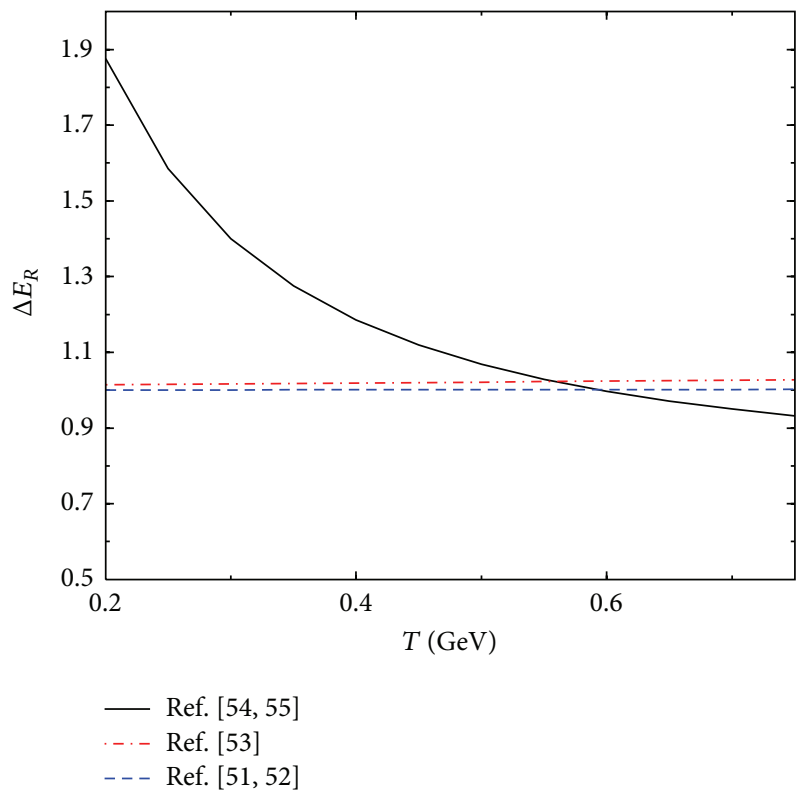

Figure 4: Temperature variation of $\Delta E_{R}$, that is, $\Delta E$ normalized by the corresponding value obtained from GB approximation, of a $15 \mathrm{GeV}$ gluon due to traversal of $4 \mathrm{fm}$ in a gluonic heat bath. Solid (dashed) line indicates result for the gluon spectrum obtained (Color online) in $[61,63]$. The dot-dashed line stands for the results for the gluon spectrum of [62].

where $\theta$ is the angle between acceleration $\dot{\vec{\beta}}$ of the particle and the direction of propagation of radiation, $\vec{n}$. This is a simple $\sin ^{2} \theta$ behaviour showing no radiation at $\theta=0$ (or $n \pi, n \epsilon$ $Z$ ). It can be shown [73] that the behaviour of (24) is, indeed, similar to what one gets for conventional dead cone [71]. Qualitatively speaking, it is hard to cause a deceleration of a high energy heavy quarks along their directions of motion, and this is why the bremsstrahlung radiation is suppressed along this direction (see Figure 24). Indeed, the distribution of radiated gluons emanating from HQs is shown to be related to those from light quarks (LQs) by the following formula [71] (for small radiation angle $\theta$ ):

$$
d P_{\mathrm{HQ}}=\left(1+\frac{\theta_{0}^{2}}{\theta^{2}}\right)^{-2} d P_{\mathrm{LQ}}
$$

where $\theta_{0}=m / E, m$ is the mass, and $E$ is the energy of heavy quark. It is worth noting that when $E \rightarrow \infty$, the radiation from HQs converges with that of LQs. However, there are some very recent developments in generalizing the dead-cone effect either from $Q q \rightarrow$ Qqg matrix element or assuming effects of off-shellness of quarks which, after being produced, take some time to become on-shell. The details may be seen in the Appendix.

The radiative transport coefficients like drag and diffusion have been evaluated in $[25,28]$ employing potential approach. The radiative drag coefficient can be obtained by finding out the radiative energy loss of HQs passing through medium. The GB spectrum for emitted gluon, weighted by the deadcone factor and the energy of gluon $k_{0}\left(=k_{\perp} \cosh \eta\right)$, is integrated over the transverse momentum $\left(k_{\perp}\right)$ and rapidity

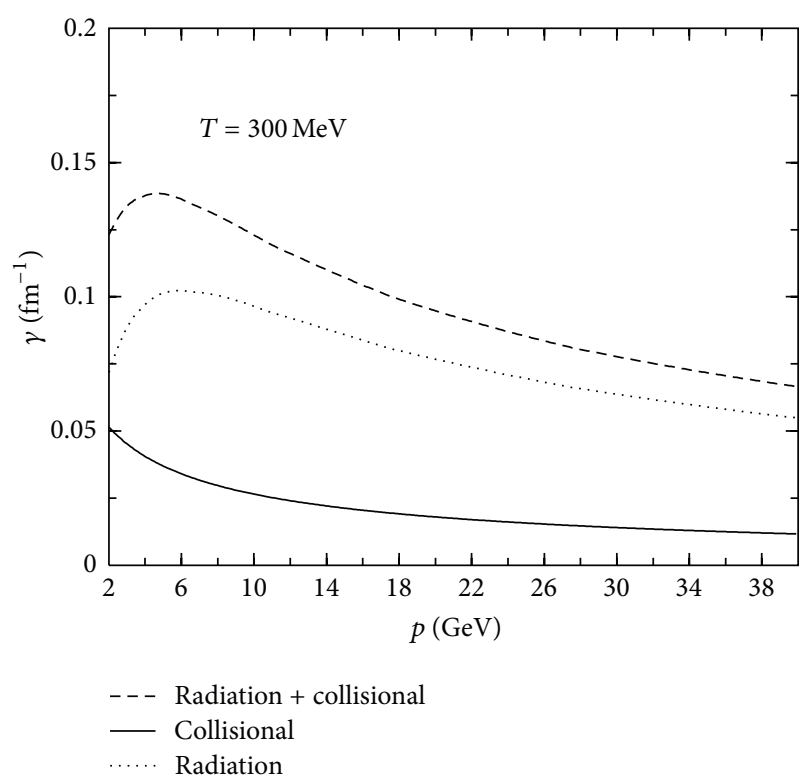

FIGURE 5: Drag coefficients of charm assuming running strong coupling, $\alpha_{s}(T)$ and temperature dependent Debye screening mass, $m_{D}(T)$ due to its interaction with thermal gluons, quarks, and antiquarks.

$(\eta)$ of the emitted gluon. As already stated, the lower and upper limits of $k_{\perp}$ have been obtained from the $\theta$-functions of (23). The average energy loss per collision, $\Delta E_{\text {rad }}$, can be written with the help of (23) as

$$
\begin{aligned}
\Delta E_{\mathrm{rad}}=\left\langle n_{g} k_{0}\right\rangle & =\int d \eta d^{2} k_{\perp} \frac{d n_{g}}{d \eta d^{2} k_{\perp}} \\
& \times k_{0} \Theta\left(\tau_{\mathrm{sc}}-\tau_{F}\right) \Theta\left(E-k_{\perp} \cosh \eta\right) F_{\mathrm{DC}}^{2}
\end{aligned}
$$

where the formation time of the emitted gluon [49], $\tau_{F}=$ $\left(C_{A} / 2 C_{2}\right) 2 \cosh \eta / k_{\perp}$, and $C_{A} / 2 C_{2}=N^{2} /\left(N^{2}-1\right)$ for quarks with $C_{2}=C_{F}=4 / 3$. Dead-cone factor of $(25)\left(d P_{\mathrm{HQ}} / d P_{\mathrm{LQ}}\right)$ can be written in the following way, provided one replaces $k_{\perp} / k_{0} \sim \sin \theta \sim \theta$ for small $\theta$ :

$$
F_{\mathrm{DC}}^{2}=\left(\frac{k_{\perp}^{2}}{k_{0}^{2} \theta_{0}^{2}+k_{\perp}^{2}}\right)^{2}
$$

where $d E / d x$ can be obtained if one multiplies $\Delta E_{\text {rad }}$ with $\Lambda$, which can be obtained from [70]. Drag $(\gamma)$ and diffusion $(D=\gamma m T)$ coefficients in [28] are functions of momentum as well as temperature. The effective drag can be obtained by adding collisional and radiative drags, $\gamma_{\text {eff }}=\gamma_{\text {coll }}+\gamma_{\text {rad }}$. The momentum dependence of the drag coefficient of the charm quark propagating through the QGP is displayed in Figure 5 for $T=300 \mathrm{MeV}$. For $p_{T} \sim 2 \mathrm{GeV}$ of charm quarks the collisional and radiative contributions tend to merge with each other. However, it is interesting to note the dominance of radiative drag in Figure 5 over its collisional counterpart for higher momentum. 


\section{Space-Time Evolution}

Once we know the total drag $\left(\gamma_{\text {eff }}\right)$ and diffusion $\left(D_{\text {eff }}\right)$ coefficients, we need the initial conditions for both the HQ and the QGP background which is also evolving with time as follows.

$$
\partial_{\mu} T^{\mu \nu}=0
$$

where $T^{\mu \nu}=(\epsilon+P) u^{\mu} u^{\nu}-g^{\mu \nu} P$ is the energy momentum tensor for ideal fluid, $\epsilon$ is the energy density, $P$ is the pressure, $u^{\mu}$ is the hydrodynamic four velocity, and $g^{\mu \nu}$ is the metric tensor. We will solve this equation for longitudinal expansion assuming boost invariance along the $z$ direction [74]. It is expected that the central rapidity region of the system formed after nuclear collisions at RHIC and LHC energy is almost net baryon free. Therefore, the equation governing the conservation of net baryon number need not be considered here. Under this circumstance, (28) reduces to:

$$
\frac{\partial \epsilon}{\partial \tau}+\frac{\epsilon+P}{\tau}=0
$$

To solve (29), we use the relation $P=c_{s}^{2} \epsilon$, where $c_{s}$ is the velocity of sound and $\tau$ is time. With this we arrive at the relation

$$
\epsilon \tau^{1+c_{s}^{2}}=C
$$

where $C$ is a constant. For the ideal equation of state, $c_{s}^{2}=$ $1 / \sqrt{3}$, (30) reads $\epsilon \tau^{4 / 3}=C$. In terms of temperature, this relation can be written as $\tau T^{3}=C$.

The total amount of energy dissipated by a heavy quark in the expanding QGP depends on the path length it traverses. Each parton traverses a different path length which depends on the geometry of the system and on the point where it is created. The probability that a parton is produced at a point $(r, \phi)$ in the plasma depends on the number of binary collisions at that point which can be taken as [75]

$$
P(r, \phi)=\frac{2}{\pi R^{2}}\left(1-\frac{r^{2}}{R^{2}}\right) \theta(R-r),
$$

where $R$ is the nuclear radius. It should be mentioned here that the expression in (31) is an approximation for the collisions with zero impact parameter. A very high energy parton created at $(r, \phi)$ in the transverse plane propagates a distance $L=\sqrt{R^{2}-r^{2} \sin ^{2} \phi}-r \cos \phi$ in the medium provided its direction remains unaltered. In the present work, we use the following equation for the geometric average of the integral involving drag coefficient

$$
\Gamma=\frac{\int r d r d \phi P(r, \phi) \int^{L / v} d \tau \gamma(\tau)}{\int r d r d \phi P(r, \phi)},
$$

where $v$ is the velocity of the propagating partons. Similar averaging has been performed for the diffusion coefficient, too. For a static system, the temperature dependence of the drag and diffusion coefficients of the heavy quarks enters via the thermal distributions of light quarks and gluons through which it is propagating. However, in the present scenario, the variation of temperature with time is governed by the equation of state or velocity of sound of the thermalized system undergoing hydrodynamic expansion. In such a scenario the quantities like $\Gamma$ (32) and hence the HQ suppression become sensitive to $c_{s}$.

\section{Initial Conditions and Nuclear Modification Factor}

In order to solve (11), the initial distribution functions, $f_{\text {in }}\left(p_{T}, t\right)$ for charm and bottom quarks have been supplied from the well-known MNR code [39]. The ratio between the solution of Fokker-Planck equation at the transition temperature, $T_{c}=175 \mathrm{MeV}$, and the initial distribution function of HQ is the required nuclear modification factor, $R_{A A}$ of open HQ. But in order to compare results from the present formalism with experimental data from RHIC and LHC, we need to have $R_{A A}$ of nonphotonic single electron originating from the decays of $D$ and $B$ mesons.

Therefore, the hadronization of charm and bottom quarks to $D$ and $B$ mesons, respectively, are done by using Peterson fragmentation function [76] as

$$
f(z) \propto \frac{1}{\left[z\left[z-1 / z-\epsilon_{\mathrm{Q}} /(1-z)\right]^{2}\right]},
$$

where $z$ is the fraction of momentum carried by the hadrons and $\epsilon_{\mathrm{Q}}$ is 0.05 for charm and $\left(m_{c} / m_{b}\right)^{2} \epsilon_{\mathrm{Q}}$ for bottom where $m_{c}\left(m_{b}\right)$ is the mass of charm (bottom). One may use different kinds of available fragmentation functions, but the final result will not be sensitive to the choice of $f(z)$. In point of fact, describing hadronization has always been a formidable task because the hadron bound states are nonperturbative in nature. The fragmentation scheme is a popular method to deal with hadronization. For more relevant information, interested readers are referred to $[77,78]$, which discusses coalescence model, a hadronization formulation based on recombination of heavy flavours.

Both the final solution of FP Equation and the initial distribution of HQ are convoluted with the above fragmentation function (33) and their ratio will give $R_{A A}$ of $D$ and $B$ mesons. The final and initial distribution functions are obtained for the single electrons originated from the decays of $D$ and $B$ mesons and the final nuclear modification factor is

$$
R_{A A}^{D(B) \rightarrow e}=\frac{f^{D(B) \rightarrow e}\left(p_{T}, T_{c}\right)}{f^{D(B) \rightarrow e}\left(p_{T}, T_{i}\right)} .
$$

Now, once we know how to determine $R_{A A}$, we will compare our results with the RHIC and LHC data. In the process of doing so, we will study the effects of the equation of state on the nuclear suppression of heavy flavours in quark gluon plasma and estimate the initial entropy density of the QGP formed at the RHIC. For this purpose, the experimental data on the charged particle multiplicity and the nuclear suppression of single electron spectra originating from the semileptonic decays of $D$ and $B$ mesons have been employed. We have 


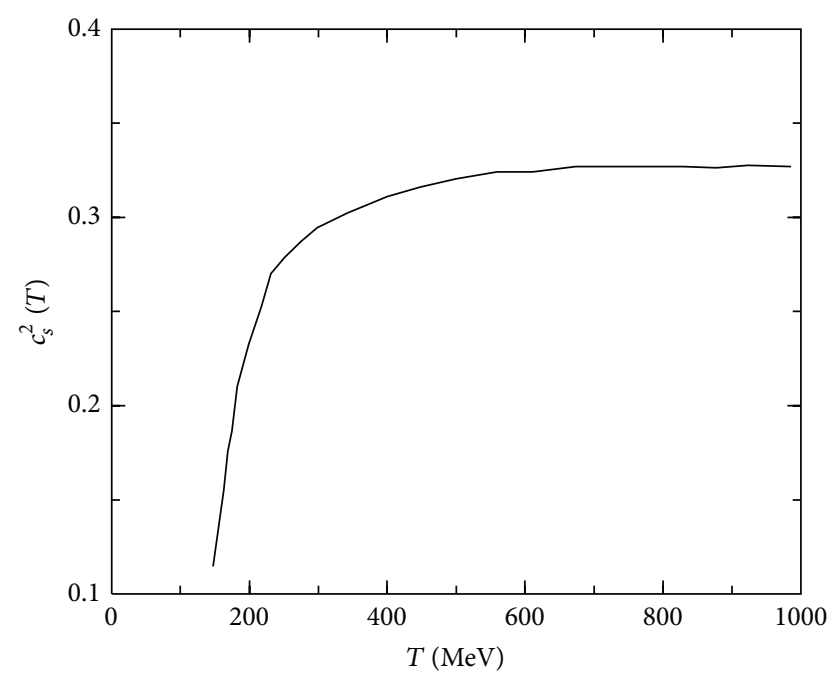

FIgURE 6: Velocity of sound squared as a function temperature [8].

used inputs from lattice QCD (LQCD) to minimize the model dependence of the results.

The initial entropy density and the thermalization time $\left(\tau_{i}\right)$ for the QGP can be constrained to the measured (final) multiplicity by the following relation [79] which is boost invariant:

$$
s_{i} \tau_{i}=\kappa \frac{1}{A_{\perp}} \frac{d N}{d y},
$$

where $A_{\perp}$ is the transverse area of the system which can be determined from the collision geometry and $\kappa$ is a known constant (=3.7 for massless Bosons).

Equation of state (EoS), which is taken as $P=c_{s}^{2} \epsilon$ for almost baryon free QGP expected at RHIC energy, also has its own role to play in the space-time evolution of QGP. This sound velocity squared appearing in the EoS shows a significant variation with temperature in LQCD calculations (Figure 6).

In Figure 10, we show the variation of $T_{i}$ with $c_{s}^{2}$ obtained by constraints imposed by the experimental data on $R_{A A}$ and $d N / d y$. The value of $T_{i}$ varies from 210 to $300 \mathrm{MeV}$ depending on the value of $c_{s}$. It is interesting to note that the lowest value of $T_{i}$ obtained from the present analysis is well above the quark-hadron phase transition temperature, indicating the fact that the system formed in $\mathrm{Au}+\mathrm{Au}$ collisions at $\sqrt{s_{N N}}=$ $200 \mathrm{GeV}$ might be formed in the partonic phase.

The data for $R_{A A}$ of $D^{0}$ from ALICE are also contrasted with our results (see Figure 11) when $c_{s}^{2}=1 / 3$ [12]. The EoS sets the expansion time scale for the system as $\tau_{\exp } \sim$ $[(1 / \epsilon) d \epsilon / d \tau]^{-1} \sim \tau /\left(1+c_{s}^{2}\right)$ indicating the fact that lower value of $c_{s}$ makes the expansion time scale longer, that is, the rate of expansion is slower. This issue is further discussed in details in [80].

The result of $R_{A A}$, where the EoS contains temperaturedependent $c_{s}^{2}$, is displayed in Figure 7. Here, the experimentally measured suppression $[13,14]$ has been reproduced reasonably well at $T_{i}=250 \mathrm{MeV}$ and $\tau_{i}=0.83 \mathrm{fm} / \mathrm{c}$. The $T_{i}$ value, however, may increase if we take into account the transverse expansion because the inflation dilutes the medium.
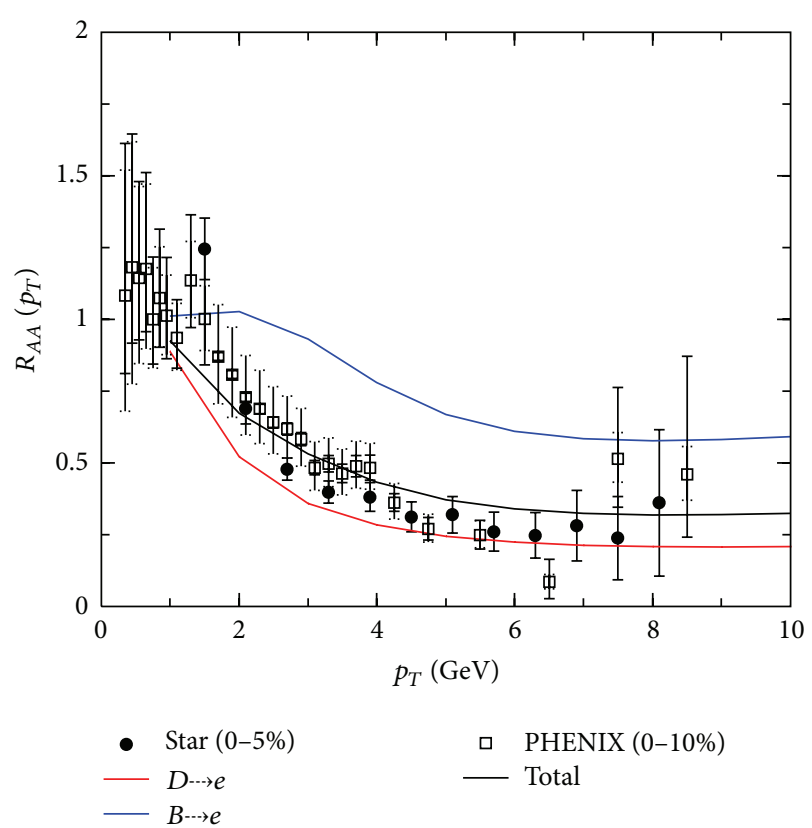

FIGURE 7: Variation of $R_{A A}$ with $p_{T}$ for the space time evolution with initial condition $T_{i}=250 \mathrm{MeV}$ and $\tau_{i}=0.84 \mathrm{fm} / \mathrm{c}$ and the EoS which includes the variation of $c_{s}$ with $T$ (colour online).

Now, we know that larger $c_{s}$ makes the QGP life time smaller leading to lesser suppression of HQ propagating through QGP for a shorter time. Therefore, when the value of $c_{s}^{2}$ is taken to be $1 / 3$, that is, the highest possible value, the maximum value of $T_{i}$, which is in this analysis $300 \mathrm{MeV}$, will be reached. The value of $s_{i}$ at $c_{s}^{2}=1 / 3$ is $\sim 59 / \mathrm{fm}^{3}$. These values of $T_{i}$ and $s_{i}$ are considered as the highest values of them admitted by the data. The result for the highest value of $c_{s}^{2}$ is illustrated in Figure 8. Likewise, the result of $R_{A A}$ in the case when $c_{s}^{2}=1 / 5$ is depicted in Figure 9. In this case, the data is well reproduced at $T_{i}=210 \mathrm{MeV}$ and $s_{i} \sim 19.66 / \mathrm{fm}^{3}$.

Now, it is expected that the central rapidity region of the system formed after nuclear collisions at high energy RHIC and LHC run is almost net baryon free. Therefore, the equation governing the conservation of net baryon number need not be considered here and all our calculations are valid for zero baryonic chemical potential $\left(\mu_{B}=0\right)$ cases. One may be interested in calculating transport coefficients in $\mu_{B} \neq 0$ case which may be of importance in low energy RHIC run $[81,82]$ and GSI-FAIR [83]. This aspect is discussed in the next section.

\section{Drag and Diffusion at Finite Baryonic Chemical Potential}

The nuclear collisions at low energy RHIC run [81, 82] and GSI-FAIR [83] are expected to create a thermal medium with large baryonic chemical potential $\left(\mu_{B}\right)$ and moderate temperature $(T)$. So the effect of baryonic chemical potential $\left(\mu_{B}\right)$ on the transport coefficients of HQ should also be taken into account. Both the temperature $(T)$ and quark chemical potential, $\mu\left(=\mu_{B} / 3\right)$, dependence of drag enter through the thermal distribution. 


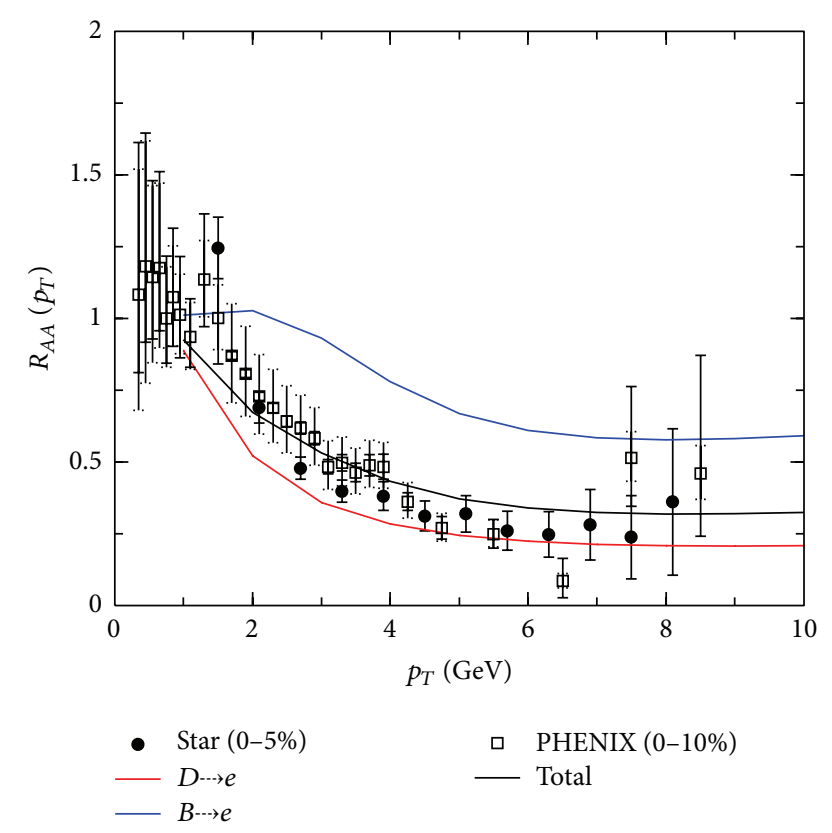

FIgURE 8: Variation of $R_{A A}$ with $p_{T}$ for for $c_{s}^{2}=1 / 3$ and $T_{i}=$ $300 \mathrm{MeV}$ (colour online).

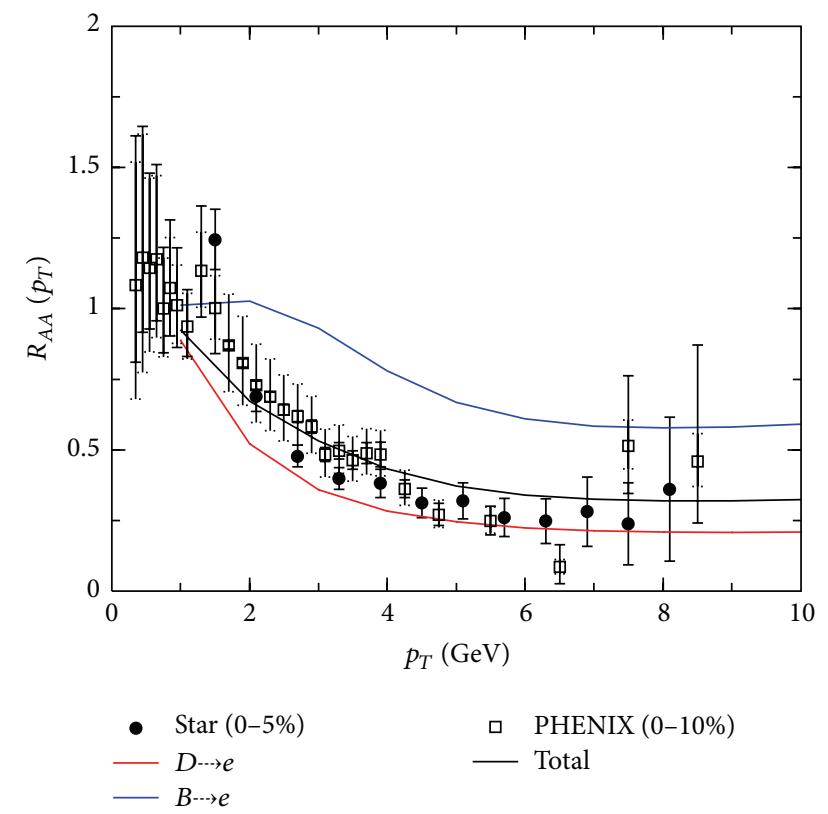

Figure 9: Variation of $R_{A A}$ with $p_{T}$ for $c_{s}^{2}=1 / 5$ and $T_{i}=210 \mathrm{MeV}$ (colour online).

The variation of the drag coefficients of charm quarks (due to its interactions with quarks and antiquarks) with the baryonic chemical potential for different $T$ is displayed in Figure 12. The drag coefficient for the process: $\mathrm{Qg} \rightarrow \mathrm{Qg}$ is $\sim 8.42 \times 10^{-3} \mathrm{fm}^{-1}\left(1.86 \times 10^{-2} \mathrm{fm}^{-1}\right)$ for $T=140 \mathrm{MeV}(190$ $\mathrm{MeV}$ ) (not displayed in Figure 12). The $T$ and $\mu$ dependence of the drag and diffusion coefficients may be understood as follows. As discussed earlier, the drag may be defined as the thermal average of the momentum transfer weighted by the square of the invariant transition amplitude for the reactions $q Q \rightarrow q Q, Q \bar{q} \rightarrow Q \bar{q}$, and $g Q \rightarrow g Q$.

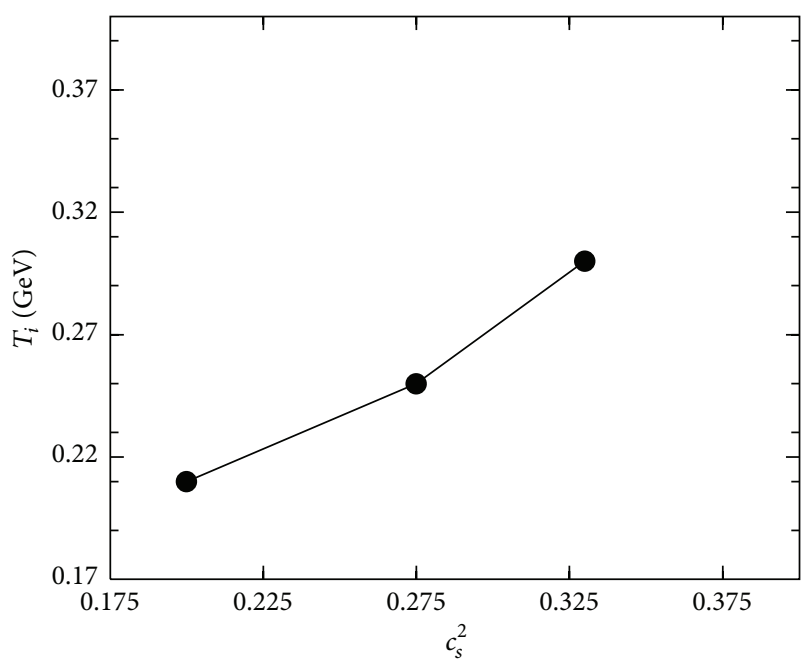

Figure 10: The variation of $T_{i}$ with $c_{s}^{2}$ for fixed $d N / d y$.

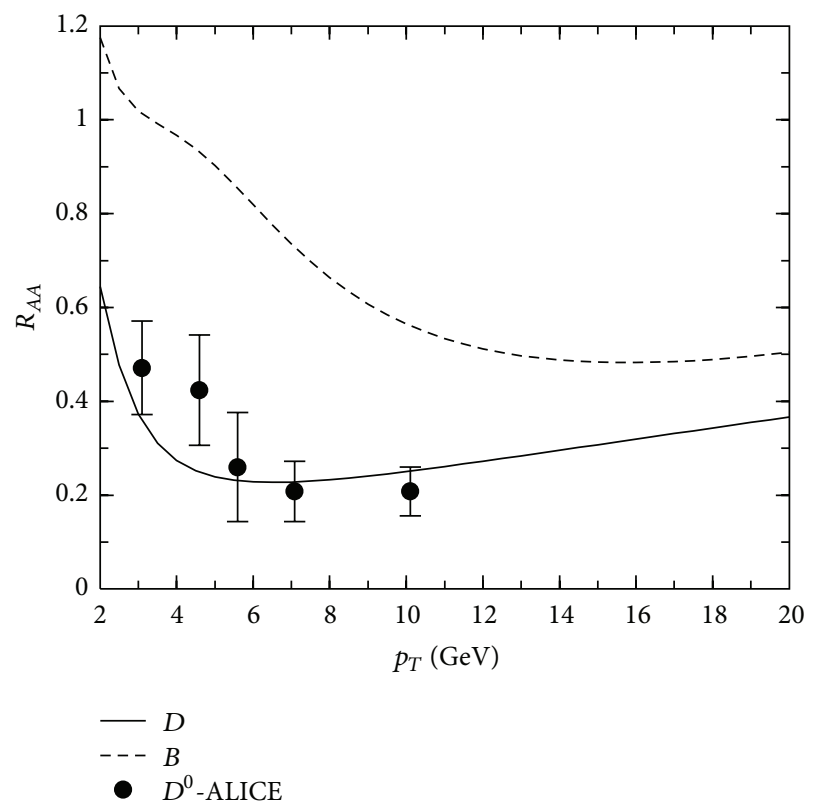

Figure 11: $R_{A A}$ as a function of $p_{T}$ for $D$ and $B$ mesons at LHC. Experimental data taken from [16].

The average momentum of the quarks of the thermal bath increases with both $T$ and $\mu$. The increase in average momenta enables the thermal quarks to transfer larger momentum and thus, in turn, enhances the drag coefficient. This trend is clearly observed in the results displayed in Figure 12 for charm quark. The drag due to the process $Q q \rightarrow Q q$ is larger than the $Q \bar{q} \rightarrow Q \bar{q}$ interaction because of non-zero chemical potential, the $Q$ propagating through which the medium encounters more $q$ than $\bar{q}$ at a given $\mu$. For vanishing chemical potential, the contributions from quarks and anti-quarks are same.

In the same way, it may be argued that the diffusion coefficient involves the square of the momentum transfer, which should also increase with $T$ and $\mu$ as observed in Figure 13. The diffusion coefficient for charm quarks due to its interaction with gluons is given by $\sim 1.42 \times 10^{-3} \mathrm{GeV}^{2} / \mathrm{fm}$ 


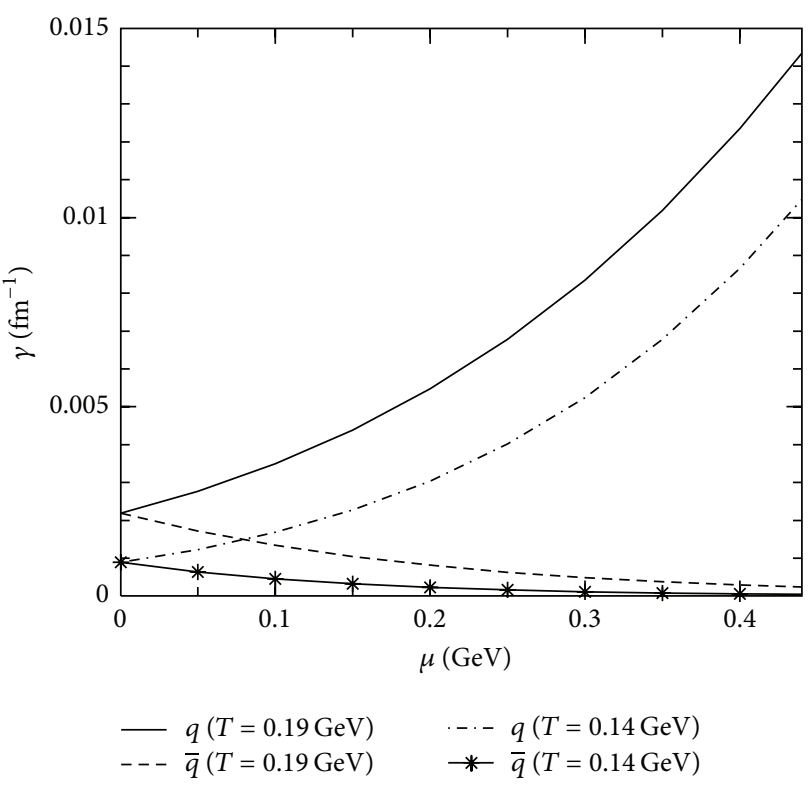

FIGURE 12: Variation of the drag coefficient of charm quark due to its interactions with light quarks and anti-quarks as a function of $\mu$ for different temperatures.

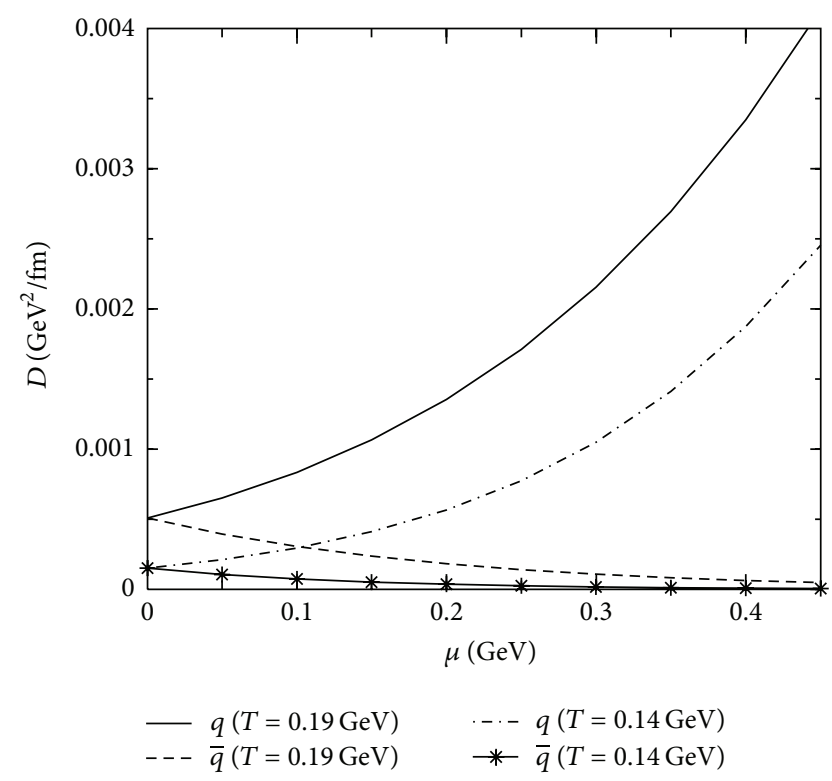

FIGURE 13: Variation of the diffusion coefficient of charm quark due to its interactions with light quarks and anti-quarks as a function of $\mu$ for different temperatures.

$\left(4.31 \times 10^{-3} \mathrm{GeV}^{2} / \mathrm{fm}\right)$ for $T=140 \mathrm{MeV}(190 \mathrm{MeV})$. The drag and diffusion coefficients for bottom quarks are displayed in Figures 14 and 15, respectively, showing qualitatively similar behaviour as charm quarks. The drag coefficients for bottom quarks due to the process $\mathrm{Qg} \rightarrow$ Qg are given by $\sim 3.15 \times$ $10^{-3} \mathrm{fm}^{-1}$ and $6.93 \times 10^{-3} \mathrm{fm}^{-1}$ at $T=140 \mathrm{MeV}$ and $190 \mathrm{MeV}$ respectively. The corresponding diffusion coefficients are $1.79 \times 10^{-3} \mathrm{GeV}^{2} / \mathrm{fm}$ and $5.38 \times 10^{-3} \mathrm{GeV}^{2} / \mathrm{fm}$ at $T=140 \mathrm{MeV}$ and $190 \mathrm{MeV}$, respectively. The $\mu$ dependent drag and diffusion coefficients will be used later to evaluate the nuclear

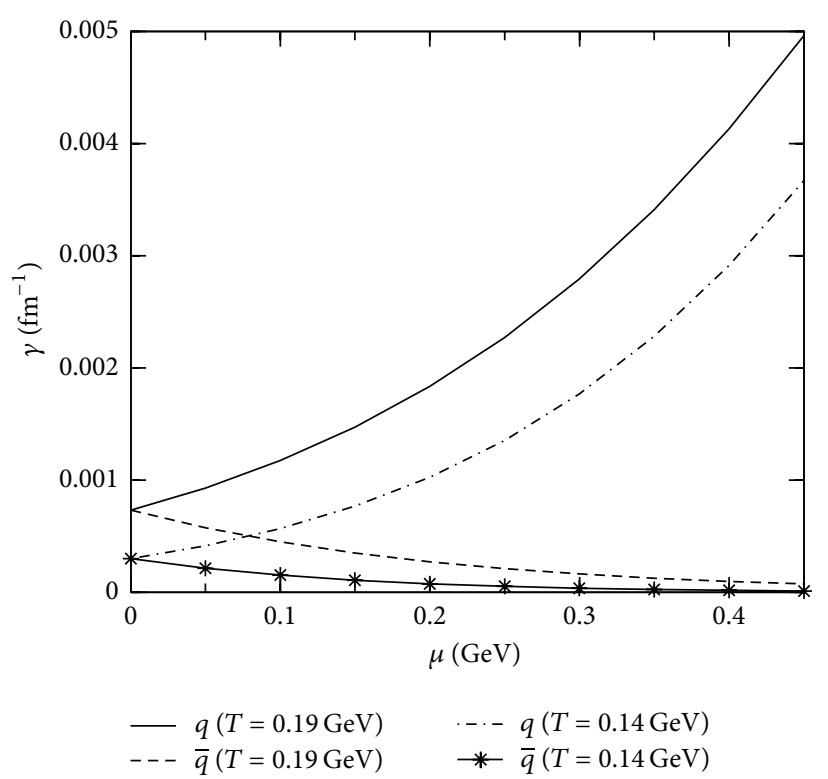

FIGURE 14: The same as Figure 12 for bottom quark.

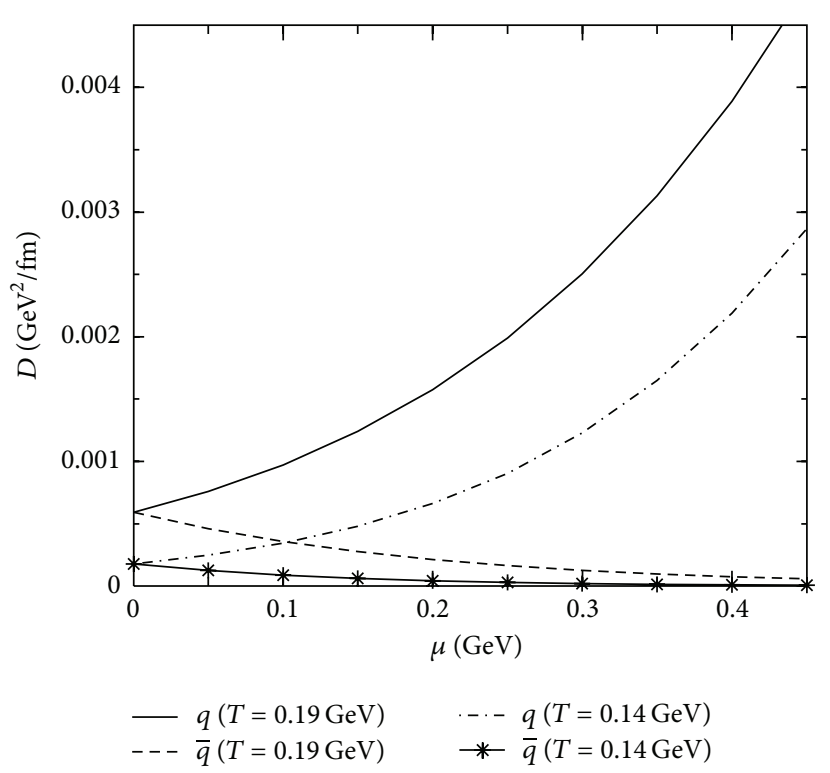

FIGURE 15: The same as Figure 13 for bottom quark.

suppression of the heavy flavours for low energy RHIC experiments.

\section{Nuclear Suppression in Baryon Rich QGP}

For low energy collisions, the radiative energy loss of heavy quarks will be much smaller than the loss caused by elastic processes as they are produced with very low momentum. Moreover, the thermal production of charm and bottom quarks can be ignored in the range of temperature and baryonic chemical potential under study. Therefore, we can apply the FP equation for the description of HQ evolution in the baryon rich QGP. Here we need to solve the FP equation for non-zero $\mu_{B}$. The drag and diffusion coefficients are functions of both the thermodynamical variables: $\mu_{B}$ and $T$. 


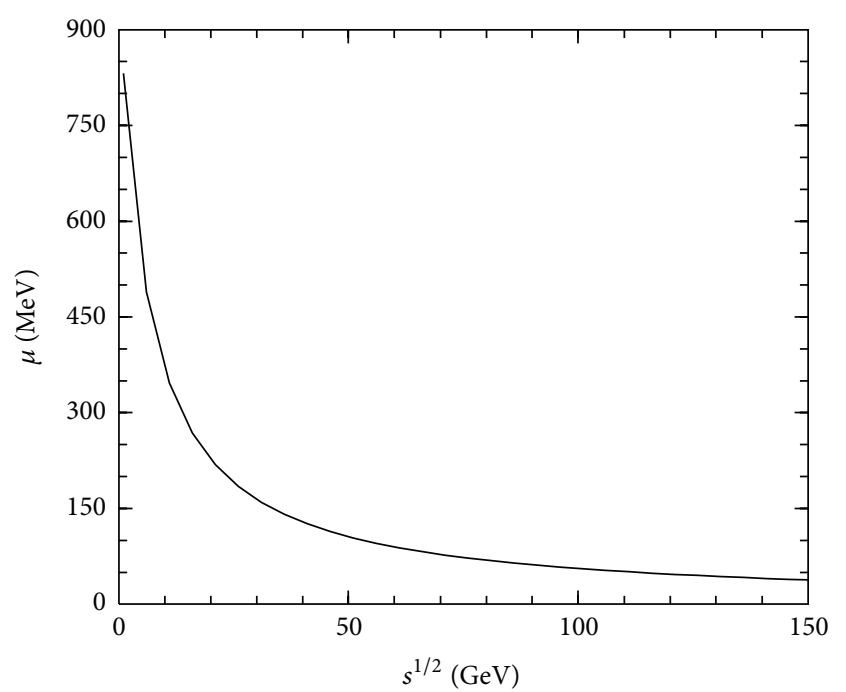

FIGURE 16: Variation of $\mu_{B}$ with respect to the $\sqrt{s_{N N}}$.

The energy $\left(\sqrt{s_{N N}}\right)$ dependence of the chemical potential can be obtained from the parametrization of the experimental data on hadronic ratios as [84] (see also [85])

$$
\mu_{B}\left(s_{N N}\right)=a\left(1+\frac{\sqrt{s_{N N}}}{b}\right)^{-1},
$$

where $a=0.967 \pm 0.032 \mathrm{GeV}$ and $b=6.138 \pm 0.399 \mathrm{GeV}$. The parametrization in (36) gives the values of $\mu_{B}$. At midrapidity, the chemical potential of the system decreases with respect to the colliding energy as observed in Figure 16. So the composition of matter produced at LHC and RHIC is different from the matter produced at low energy collision. At LHC and RHIC, the matter produced at the midrapidity is almost baryon free, whereas the matter produced at the low colliding energy is dominated by baryons.

To take care of this extra baryons, we need to solve the baryon-number conservation equation along with the energy-momentum conservation equation; that is, we simultaneously solve

$$
\begin{gathered}
\partial_{\mu} T^{\mu \nu}=0, \\
\partial_{\mu} n_{B}^{\mu}=0
\end{gathered}
$$

for $(1+1)$ dimension with boost invariance along the longitudinal direction [74]. In the above equation, $n_{B}^{\mu}=n_{B} u^{\mu}$ is the baryonic flux and $u^{\mu}$ is the hydrodynamic 4 -velocity. The initial baryonic chemical potential carried by the quarks $\mu\left(=\mu_{B} / 3\right)$ is shown in Table 1 for various $\sqrt{s_{N N}}$ under consideration.

The value of the multiplicities for various $\sqrt{s_{N N}}$ has been calculated from the equation below [86] as

$$
\frac{d N}{d y}=\frac{d n_{\mathrm{pp}}}{d y}\left[(1-x) \frac{\left\langle N_{\text {part }}\right\rangle}{2}+x\left\langle N_{\text {coll }}\right\rangle\right]
$$

$N_{\text {coll }}$ is the number of collisions and contributes $x$ fraction to the multiplicity $d n_{\mathrm{pp}} / d y$ measured in pp collision. The
TABLE 1: The values center of mass energy $\sqrt{s_{N N}}, d N / d y$, initial temperature $\left(T_{i}\right)$, and quark chemical potential used in the present calculations.

\begin{tabular}{lccc}
\hline$\sqrt{\left(s_{N N}\right)}(\mathrm{GeV})$ & $d N / d y$ & $T_{i}(\mathrm{MeV})$ & $\mu(\mathrm{MeV})$ \\
\hline 39 & 617 & 240 & 62 \\
27 & 592 & 199 & 70 \\
17.3 & 574 & 198 & 100 \\
7.7 & 561 & 197 & 165 \\
\hline
\end{tabular}

number of participants, $N_{\text {part }}$, contributes a fraction $(1-x)$ to $d n_{\mathrm{pp}} / d y$, which is given by

$$
\frac{d n_{\mathrm{pp}}}{d y}=2.5-0.25 \ln (s)+0.023 \ln ^{2}(s) \text {. }
$$

The values of $N_{\text {part }}$ and $N_{\text {coll }}$ are estimated for $(0 \%-5 \%)$ centrality by using Glauber model [87]. The value of $x$ depends very weakly on $\sqrt{s_{N N}}$ [88], and in the present work we have taken $x=0.1$ for all the energies.

We need the initial heavy quark momentum distributions for solving the FP equation. For low collision energy, rigorous QCD-based calculations for heavy flavour production are not available. In the present work, the initial HQ distribution is obtained from pQCD calculation [41, 89] for the processes $g g \rightarrow Q \bar{Q}$ and $q \bar{q} \rightarrow Q \bar{Q}$. To demonstrate the effect of nonzero baryonic chemical potential, we evaluate $R_{A A}$ for $\mu=$ $200 \mathrm{MeV}$ and $\mu=0$ for a given $T_{i}=200 \mathrm{MeV}$. The results are displayed in Figure 17 representing the combined effects of temperature and baryon density on the drag and diffusion coefficients. The drag of the heavy quarks due to its interaction with quarks is larger than that due to its interactions with the anti-quarks (Figure 12), resulting in larger suppression in the former case than the latter. The net suppression of the electron spectra from the $\mathrm{Au}+\mathrm{Au}$ collisions compared to $\mathrm{p}+\mathrm{p}$ collisions is affected by quarks, anti-quarks, and gluons. The results for net suppressions are displayed for $\mu=200 \mathrm{MeV}$ (dashed line) and $\mu=0$ (with asterisk). The experimental detection of the non-zero baryonic effects will shed light on the net baryon density (and hence baryon stopping) in the central rapidity region. However, whether the effects of nonzero baryonic chemical potential are detectable or not will depend on the overall experimental performance.

The results for $R_{A A}$ are shown in Figure 18 for various $\sqrt{s_{N N}}$ with inputs from Table 1 . We observe that at large $p_{T}$ the suppression is similar for all energies under consideration. This is because the collisions at high $\sqrt{s_{N N}}$ are associated with large temperature but small baryon density at midrapidity, which is compensated by large baryon density and small temperature at low $\sqrt{s_{N N}}$ collisions. Low $p_{T}$ particles predominantly originate from low temperature and low density part of the evolution where drag is less and so is the nuclear suppression.

In our earlier work [23], we have evaluated the $R_{A A}$ for nonphotonic single electron spectra resulting from the semileptonic decays of hadrons containing heavy flavours and observed that the data from RHIC collisions at $\sqrt{s_{N N}}=$ $200 \mathrm{GeV}$ are well reproduced by enhancing the pQCD cross 


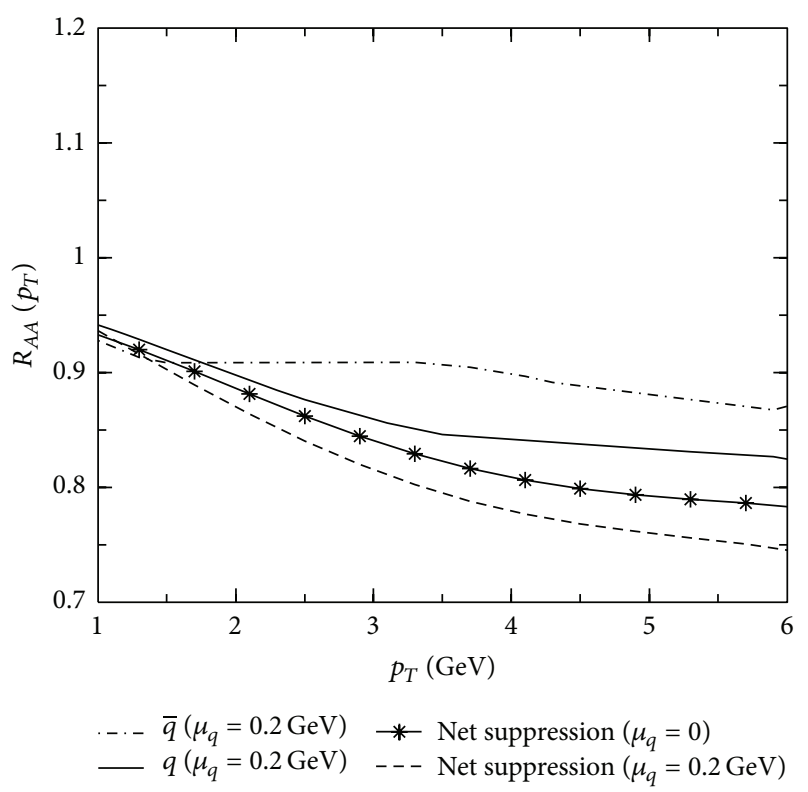

FIGURE 17: The nuclear suppression factor $R_{A A}$ as a function of $p_{T}$ due to the interaction of the charm quark (solid line) and anti-quark (dashed-dot line) for $\mu=200 \mathrm{MeV}$. The net suppressions including the interaction of quarks, anti-quarks, and gluons for $\mu=200 \mathrm{MeV}$ (dashed line) and $\mu=0$ (with asterisk) are also shown.

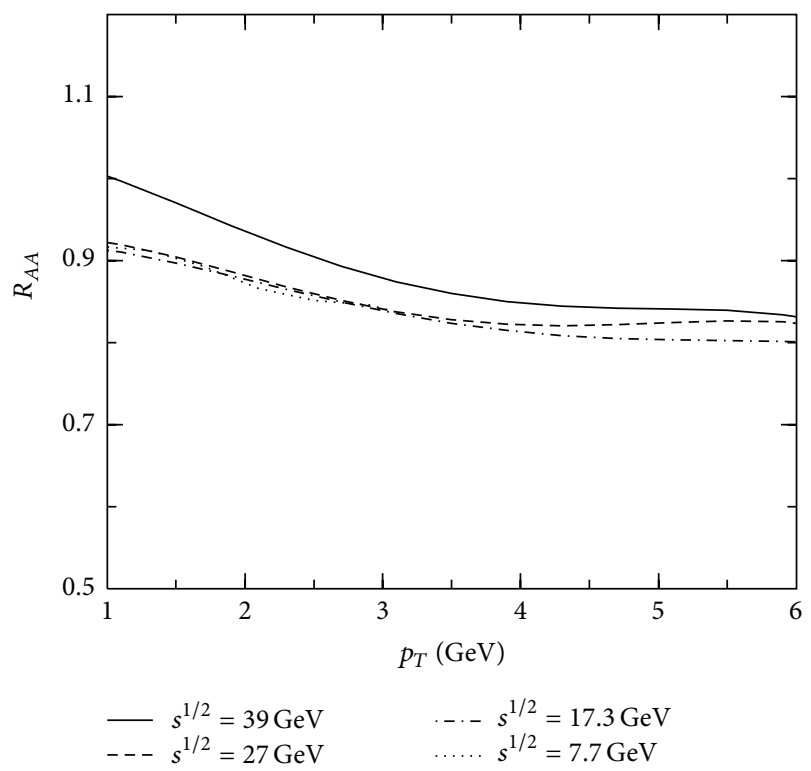

Figure 18: Nuclear suppression factor, $R_{A A}$, as function of $p_{T}$ for various $\sqrt{s_{N N}}$.

sections by a factor 2 and with an equation of state $P=\epsilon / 4$ for collisional loss. In the same spirit, we evaluate $R_{A A}$ with twice enhanced PQCD cross section and keeping all other quantities unaltered (Figure 19). The results in Figure 19 show stronger suppression as compared to the results displayed in Figure 18, but it is similar in all the energies under consideration. When we have enhanced the $\mathrm{PQCD}$ cross section for the interaction of the heavy quarks with the thermal system by a factor of two-the resulting suppressions in $R_{A A}$ are between $20 \%$ and $30 \%$ for $\sqrt{s_{N N}}=39-7.7 \mathrm{GeV}$.

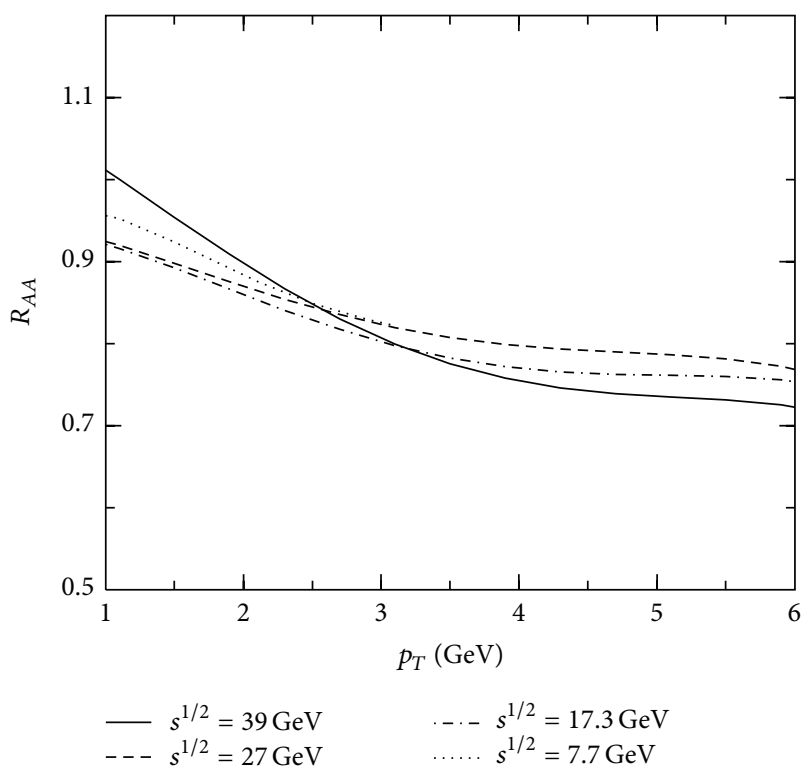

FIgURE 19: The same as Figure 18 with enhancement of cross section by a factor of 2 .

\section{Glimpses of Elliptic Flow}

However, apart from the nuclear suppression factor $R_{A A}$, another experimental observable of heavy flavour, elliptic flow $\left(v_{2}\right)$, can be studied within the framework of FokkerPlanck equation. The elliptic flow $\left(v_{2}\right)$ of the produced particles has been considered as one of the most promising signals for the early thermalization of the matter formed in heavy ion collision. If we consider a thermalized ellipsoidal spatial domain of QGP, originated due to noncentral nucleus nucleus collisions, with major and minor axes of lengths $l_{y}$ and $l_{x}$ (determined by the collision geometry), respectively, then the pressure gradient is larger along the minor axis compared to that along the major axis because $l_{y}>l_{x}$. Now, pressure gradient is force and hence force along the minor axis is larger than that along the minor axis. Consequently, the HQ moves faster in this direction. Therefore, the momentum distribution of electrons originating from the decays of charmed hadrons ( $D$ mesons) produced from the charm quark fragmentation will be anisotropic, and since $v_{2}$ is the second Fourier's coefficient of the momentum distribution, the spatial anisotropy is thus reflected in the momentum space anisotropy.

$v_{2}$ is sensitive to the initial conditions and the equation of state (EoS) of the evolving matter formed in heavy ion collision [90-92]. Several theoretical attempts have been made in this direction to study both the nuclear suppression factor and elliptic flow of the heavy flavour within a single framework. Although several authors have reproduced the large suppression of high momentum heavy quarks reasonably well yet it is still difficult to explain HQ elliptic flow simultaneously within the same set of initial parameters. It is also imperative to mention that no calculation with radiative energy loss is still able to reproduce heavy flavour elliptic flow. After this brief discussion, we refer the interested readers 
to $[18,27,32,33,56,93-96]$ for more interesting aspects of elliptic flow.

\section{Summary and Discussions}

In summary, we have tried to give a systematic account of evaluating collisional and radiative transport coefficients of heavy quarks passing through quark-gluon plasma. The nuclear suppression factor $\left(R_{A A}\right)$ of heavy quarks has been evaluated using Fokker-Planck equation. The present paper confines the discussions within perturbative QCD. Recently, the authors of [97] find out the drag force and $R_{A A}$ of charm quarks propagating through thermalized QGP within the framework of both AdS/CFT and AdS/non-CFT. Interested readers are referred to $[98,99]$ for similar discussions.

We have studied the momentum dependence of transport coefficients and observe that the momentum dependence is crucial in reproducing the trend in the $p_{T}$ dependence of the experimental data. Also, the dead-cone factor $\left(F_{\mathrm{DC}}\right)$ weighted by the Gunion-Bertsch spectrum for radiated gluon from heavy quarks is implemented. It is worth mentioning that though the present paper speaks at length about elastic as well as bremsstrahlung energy loss of HQs and tells about dominance of the latter in high $p_{T}$ region, yet there are a plethora of articles which show the importance of elastic energy loss in context of experimental observables in entire momentum range attainable by HQs $[18,93,100]$. This issue, as a matter of fact, still awaits an unambiguous settlement.

Momentum independent transport coefficients in nonzero baryonic chemical potential region have also been studied and theoretical estimates of the suppression factor are provided. In the Appendix, we have discussed the calculation of elastic transport coefficients within the ambit of QCD hard thermal loop perturbation theory. Also, radiative suppression due to off-shellness of produced partons, which we may call the "dead cone due to virtuality," has also been discussed. This effect of off-shellness of heavy quarks as well as HTL calculations may be extended to encompass radiative processes which, as we have already discussed, will play the most significant part in high energy regime. The calculations of $R_{A A}$ including the aspects just mentioned may be contrasted with the experimental data.

Though $[18,23-25,27,34,56,93,95]$ attempt to study $R_{\text {AA }}$ and $v_{2}$ of the heavy quark, yet the role of hadronic matter has been ignored. However, to make the characterization of QGP reliable, the role of the hadronic phase should be taken into consideration and its contribution must be subtracted out from the observables. Although a large amount of work has been done on the diffusion of heavy quarks in QGP, the diffusion of heavy mesons in hadronic matter has received much less attention so far. Recently, the diffusion coefficient of $D$ meson has been evaluated using heavy meson chiral perturbation theory [101] and also by using the empirical elastic scattering amplitudes [102] of $D$ mesons with thermal hadrons. The interactions of $D$ meson with pions, nucleons, kaons, and eta particles have been evaluated using Born amplitudes [103] and unitarized chiral effective $D \pi$ interactions $[104,105] . D$ and $B$-meson scattering lengths have also been used as dynamical input to study the drag and diffusion coefficients [106, 107]. All these studies observed that the magnitude of both the transport coefficients is significant, indicating substantial amount of interaction of the heavy mesons with the thermal bath. The results may have significant impact on the experimental observables like the suppression of single electron spectra [108] originating from the decays of heavy mesons produced in nuclear collisions at RHIC and LHC energies.

\section{Appendix}

\section{A. Hard Thermal Loop (HTL) Approximations and Transport Coefficients}

We have used $T=0$ pQCD matrix elements for calculating collisional transport coefficients so far. The $t$ channel divergence due to soft intermediary gluon exchange has been shielded by an ad hoc replacement of $t$ by $t-m_{D}^{2}$, where $m_{D}$ is the (static) Debye screening mass of gluon. Here we use thermal resumed (HTL) gluon propagators $[66,67,109]$ for calculating the matrix elements for the processes like $\mathrm{Qq} \rightarrow$ $Q q$ and $Q g \rightarrow Q g$, where $Q(q)$ stands for heavy (light) quark and $g$ stands for gluon, for a self-consistent shielding of $t$ channel divergence. Since the light quarks and gluons of thermal bath are hard, that is, their momenta are $\sim T$, we may neglect the vertex correction (assuming $g \ll 1$ ) arising due to ggg or qqg vertex. The effect of full gluon spectral function on the collisional drag coefficient will be investigated for HQs.

\section{B. HTL Gluon Propagator}

For finding out the HTL gluon propagator $\left(\Delta^{\mu \nu}\right)$, we need HTL approximated self-energy of gluon which goes as an input to $\Delta^{\mu \nu}$ to be used as effective thermal propagator regularizing the $t$ channel divergence. The gluon self-energy in HTL approximation is discussed in detail in $[66,67]$. In this section, we give only an outline of the scheme. There are four diagrams which contribute to gluon self-energy (Figure 20). The loop integrations can be written down easily if we keep in mind that the loop momentum, $K=\left(k_{0}, \vec{k}\right)$, is much larger compared to external gluon momentum, that is, $K \gg P$, which enables us to use simplified ggg vertex [66]. Our goal will be to find out $T^{2}$ contributions of self-energy which is the leading behaviour of self-energy in terms of temperature, $T$. This $T^{2}$ contribution of self-energy is called the "hard thermal loop" contribution. We may argue that the hard thermal loop contribution of the gluon self-energy is due to momenta $\sim T$ if we look at the generic integral of the type

$$
\int_{0}^{\infty} k f(k) d k=\frac{\pi^{2} T^{2}}{12}
$$

which appears during self-energy calculation. $f$ in (B.1) is the distribution function and the leading contribution in (B.1) is given by $k \sim T$. Henceforth, we obtain a scale of momentum $\sim T$, which will be called "hard" compared to another "soft" scale $\sim g T$, where $g(\ll 1)$ is the colour charge. HTL gluon propagators are to be used for processes exchanging very soft 


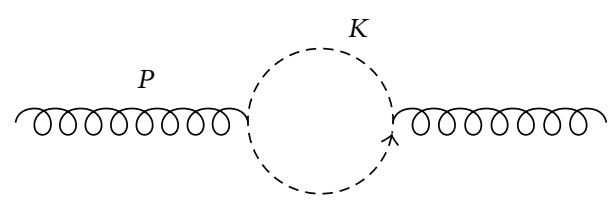

(a)

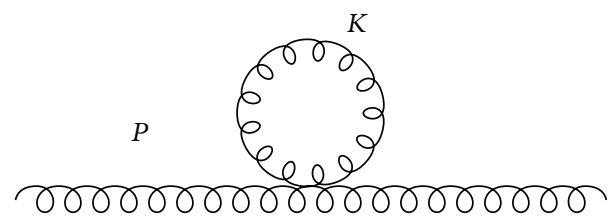

(b)

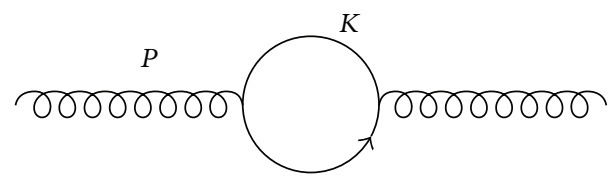

(c)

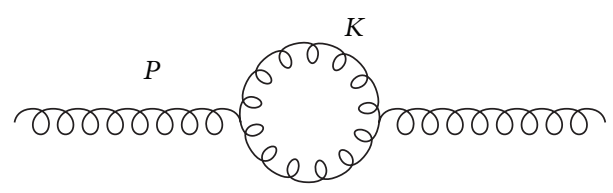

(d)

FIGURE 20: Feynman diagrams contributing to gluon self-energy up to one loop. (a) Ghost-gluon loop. (b) Four-gluon vertex. (c) Quarkantiquark pair creation. (d) Three-gluon vertex.

gluons. As the $t$ channel divergence is equivalent to dominance of soft gluon exchange processes, the use of HTL propagator is justified and consistent.

Now, let us define the following useful quantities [66] required to write down the gluon propagator in thermal medium. Let $u_{\mu}$ be the fluid 4-velocity, with normalization condition $u^{\mu} u_{\mu}=1$. The fluid 4-velocity gives rise to two directions, and so any 4 -vector $P^{\mu}$ can be decomposed into components parallel and perpendicular to the fluid velocity

$$
\begin{gathered}
\omega=P \cdot u, \\
\widetilde{P}_{\mu}=P_{\mu}-u_{\mu}(P \cdot u),
\end{gathered}
$$

where

$$
\begin{gathered}
P^{2}=\omega^{2}-p^{2}, \\
\widetilde{P}^{2}=-p^{2} .
\end{gathered}
$$

Equations (B.2) and (B.3) are valid in the local rest frame of fluid, that is, in a frame where $u=(1, \overrightarrow{0})$. Similarly, a tensor orthogonal to $u_{\mu}$ can be defined as

$$
\widetilde{g}_{\mu \nu}=g_{\mu \nu}-u_{\mu} u_{\nu}
$$

The longitudinal and transverse projection tensors, $\mathscr{P}_{L}^{\mu \nu}$ and $\mathscr{P}_{T}^{\mu \nu}$, respectively, are defined as [109]

$$
\begin{gathered}
\mathscr{P}_{L}^{\mu \nu}=-\frac{1}{P^{2} p^{2}}\left(\omega P^{\mu}-P^{2} u^{\mu}\right)\left(\omega P^{\nu}-P^{2} u^{\nu}\right), \\
\mathscr{P}_{T}^{\mu \nu}=\widetilde{g}_{\mu \nu}+\frac{\widetilde{P}_{\mu} \widetilde{P}_{\nu}}{p^{2}}
\end{gathered}
$$

which are orthogonal to $P^{\mu}$ as well as to each other, that is,

$$
P_{\mu} \mathscr{P}_{L}^{\mu \nu}=P_{\mu} \mathscr{P}_{T}^{\mu \nu}=\mathscr{P}_{L \nu}^{\mu} \mathscr{P}_{T}^{\nu \rho}=0
$$

but

$$
\mathscr{P}_{i}^{\mu \rho} \mathscr{P}_{i v \rho}=\mathscr{P}_{i v}^{\mu}, \quad i=L, T .
$$

The HTL gluon propagator, which is given by

$$
\Delta^{\mu \nu}=\frac{\mathscr{P}_{T}^{\mu \nu}}{-P^{2}+\Pi_{T}}+\frac{\mathscr{P}_{L}^{\mu \nu}}{-P^{2}+\Pi_{L}}+(\alpha-1) \frac{P^{\mu} P^{\nu}}{P^{2}},
$$

will need HTL approximated longitudinal and transverse selfenergies $\Pi_{L}$ and $\Pi_{T}$, respectively, too. $\Pi_{L}$ and $\Pi_{T}$ are given by

$$
\Pi_{L}(P)=\left(1-x^{2}\right) \pi_{L}(x), \quad \Pi_{T}(P)=\pi_{T}(x),
$$

where $x=\omega /|\vec{p}|(P \equiv(\omega, \vec{p})$, see Figure 20) and scaled selfenergies $\pi_{T}$ and $\pi_{L}$ are given by [66]

$$
\begin{gathered}
\pi_{T}(x)=m_{D}^{2}\left[\frac{x^{2}}{2}+\frac{x}{4}\left(1-x^{2}\right) \ln \left(\frac{1+x}{1-x}\right)-i \frac{\pi}{4} x\left(1-x^{2}\right)\right], \\
\pi_{L}(x)=m_{D}^{2}\left[1-\frac{x}{2} \ln \left(\frac{1+x}{1-x}\right)+i \frac{\pi}{2} x\right]
\end{gathered}
$$

where $m_{D}$ is the thermal mass of gluon and is given by $m_{D}^{2}=$ $g^{2} T^{2}\left(C_{A}+N_{f} / 2\right) / 6$, where $C_{A}=3$ is the Casimir of adjoint representation of SU(3) and $N_{f}=2$ is the number of flavours.

\section{Finding Out Matrix Elements for Qq Scattering}

From Figure 21, we can calculate the $t$-channel matrix element for the process $Q q \rightarrow Q q$. We will use the HTL gluon propagator [66]. Pictorially, an HTL propagator will be denoted by a solid circle. We can write the amplitude in Feynman Gauge $(\alpha=1)$ from Figure 21 as

$$
\begin{aligned}
-i M_{t}= & \bar{u}\left(p_{3}\right)\left(-i g \gamma^{\mu} t_{j i}^{a}\right) u\left(p_{1}\right)\left[-i \Delta_{\mu \nu}\right] \\
& \times \bar{u}\left(p_{4}\right)\left(-i g \gamma^{\nu} t_{l k}^{a}\right) u\left(p_{2}\right),
\end{aligned}
$$

where $g$ is strong coupling and $g^{2}=4 \pi \alpha_{s} . i, j, k, l(i \neq j, k \neq l)$ are quark colours and " $a$ " is the colour of intermediary gluon 


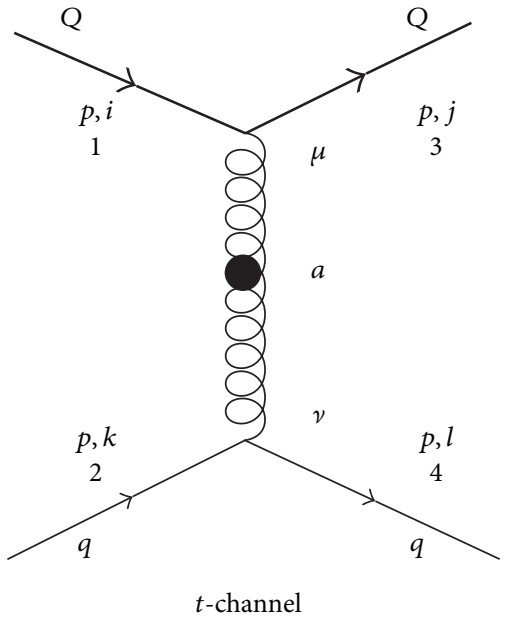

Figure 21: $Q q \rightarrow Q q$ Feynman diagram. Bold lines are for heavy quarks $(Q)$.

with polarizations $\mu, \nu$. After squaring and averaging over spin and colour as well as using (B.8), we get

$$
\begin{aligned}
\frac{\left|M_{\mathrm{Qq}}\right|^{2}}{4 C_{\mathrm{Qq}} g^{4}} & =2 \frac{p_{4} \cdot \mathscr{P}_{T} \cdot p_{3} p_{2} \cdot \mathscr{P}_{T} \cdot p_{1}}{\left(t-\Pi_{T}\right)^{2}}+2 \frac{p_{4} \cdot \mathscr{P}_{L} \cdot p_{3} p_{2} \cdot \mathscr{P}_{L} \cdot p_{1}}{\left(t-\Pi_{L}\right)^{2}} \\
& +2 \frac{p_{4} \cdot \mathscr{P}_{T} \cdot p_{1} p_{2} \cdot \mathscr{P}_{T} \cdot p_{3}}{\left(t-\Pi_{T}\right)^{2}}+2 \frac{p_{4} \cdot \mathscr{P}_{L} \cdot p_{1} p_{2} \cdot \mathscr{P}_{L} \cdot p_{3}}{\left(t-\Pi_{L}\right)^{2}} \\
& +2 A \frac{p_{4} \cdot \mathscr{P}_{L} \cdot p_{3} p_{2} \cdot \mathscr{P}_{T} \cdot p_{1}+p_{4} \cdot \mathscr{P}_{T} \cdot p_{3} p_{2} \cdot \mathscr{P}_{L} \cdot p_{1}}{\left(t-\Pi_{T}\right)^{2}\left(t-\Pi_{L}\right)^{2}} \\
& +2 A \frac{p_{4} \cdot \mathscr{P}_{L} \cdot p_{1} p_{2} \cdot \mathscr{P}_{T} \cdot p_{3}+p_{4} \cdot \mathscr{P}_{T} \cdot p_{1} p_{2} \cdot \mathscr{P}_{L} \cdot p_{3}}{\left(t-\Pi_{T}\right)^{2}\left(t-\Pi_{L}\right)^{2}} \\
& -2 p_{4} \cdot p_{2}\left[\frac{p_{3} \cdot \mathscr{P}_{T} \cdot p_{1}}{\left(t-\Pi_{T}\right)^{2}}+\frac{p_{3} \cdot \mathscr{P}_{L} \cdot p_{1}}{\left(t-\Pi_{L}\right)^{2}}\right] \\
& -m^{2}\left[2 \frac{p_{4} \cdot p_{2}}{\left(t-\Pi_{T}\right)^{2}}+\frac{p_{4} \cdot p_{2}}{\left(t-\Pi_{L}\right)^{2}}\right], \\
& +2 p_{3} \cdot p_{1}\left[\frac{p_{4} \cdot \mathscr{P}_{T} \cdot p_{2}}{\left(t-\Pi_{T}\right)^{2}}+\frac{p_{4} \cdot \mathscr{P}_{L} \cdot p_{2}}{\left(t-\Pi_{L}\right)^{2}}\right] \\
& +p_{3} \cdot p_{1}\left[2 \frac{p_{4} \cdot \mathscr{P}_{T} \cdot p_{2}}{\left(t-\Pi_{T}\right)^{2}}+2 \frac{p_{4} \cdot \mathscr{P}_{L} \cdot p_{2}}{\left(t-\Pi_{L}\right)^{2}}\right] \\
& \left.=\frac{1}{\left(t-\Pi_{L}\right)^{2}}\right]
\end{aligned}
$$

where $C_{Q q}=2 / 9$ is the Color factor, $Q^{2} \equiv t=\left(p_{1}-p_{3}\right)^{2}$, and $A=t^{2}-t\left(\operatorname{Re} \Pi_{T}+\operatorname{Re} \Pi_{L}\right)+\operatorname{Re} \Pi_{T} \Pi_{L}^{*}$, and we have used the following relations:

$$
p_{1} \cdot \mathscr{P}_{L} \cdot p_{2}=p_{3} \cdot \mathscr{P}_{L} \cdot p_{4}=p_{4} \cdot \mathscr{P}_{L} \cdot p_{1}=p_{2} \cdot \mathscr{P}_{L} \cdot p_{3}
$$

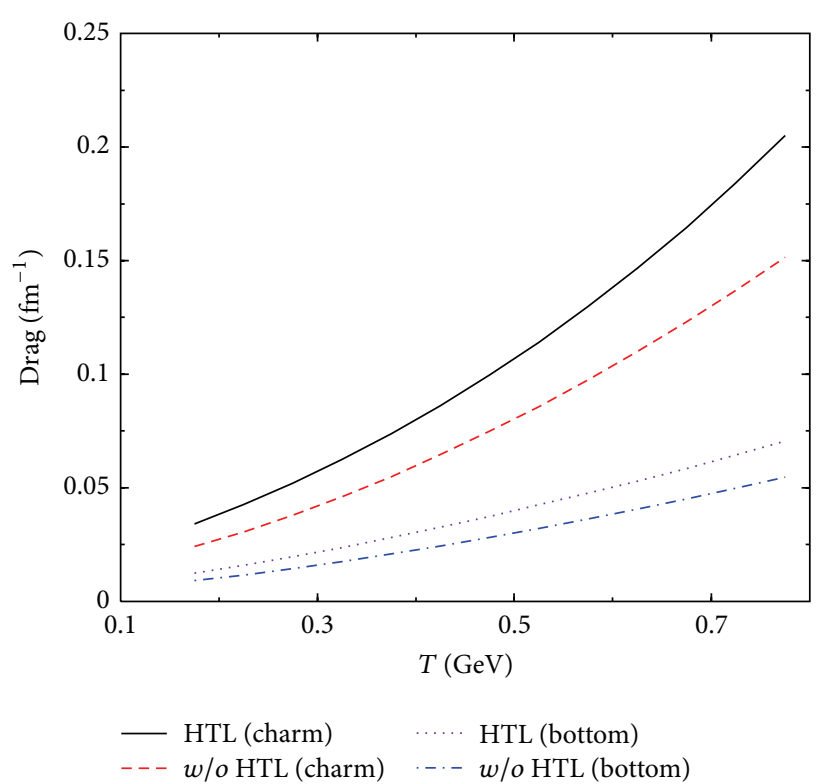

FIGURE 22: Variation of drag of heavy quarks of momentum $1 \mathrm{GeV}$ with temperature (color online).

as well as

$$
\begin{gathered}
\Delta^{\mu \rho} \Delta_{\rho}^{* \nu}=\frac{\mathscr{P}_{T}^{\mu \nu}}{\left(t-\Pi_{T}\right)^{2}}+\frac{\mathscr{P}_{L}^{\mu \nu}}{\left(t-\Pi_{L}\right)^{2}}, \\
|\Delta|^{2}=\Delta^{\mu \nu} \Delta_{\gamma_{\mu}}^{*}=\frac{2}{\left(t-\Pi_{T}\right)^{2}}+\frac{1}{\left(t-\Pi_{L}\right)^{2}}
\end{gathered}
$$

which can be proven using (B.5) and (B.8) in the rest frame of fluid element.

The $Q g \rightarrow Q g$ scattering contains $s, t$, and $u$ channel diagrams and they can easily be written just like $Q q$ case. There are $\left|M_{t}\right|^{2},\left|M_{s}\right|^{2}$ and $\left|M_{u}\right|^{2}$ as well as the interference of $s, t, u$ channels as opposed to [47] where only $s$ and $u$ channel interference term exists (besides $\left|M_{t}\right|^{2}$ ) because gluon momenta are assumed to be much larger than that of HQ.

\section{Results and Discussions on Collisional Drag and Diffusion Coefficients Using HTL Propagator}

We have found out the matrix elements for relativistic heavy quark scattering elastically with light quarks and gluons of QGP with arbitrary scattering angle. The variations of drags with temperature for HQs with momentum, $p=1 \mathrm{GeV}$, as a function of temperature are displayed in Figure 22. The results clearly indicate an enhancement and rapid variation of drag using HTL propagator $\left(\gamma_{\mathrm{HTL}}\right)$ compared to that in $T=0$ pQCD $(\gamma)$. The increase is more prominent for charm than bottom. We have explicitly checked that, in the static limit, the drag and diffusion using HTL propagator approaches that in $T=0$ pQCD. $\gamma_{\mathrm{HTL}}$ is greater than $\gamma$ for the entire momentum range considered here. Again, drag being the measure of energy loss, increase in drag results in 
more suppression of heavy flavours measured at RHIC and LHC energies. From Figure 22, we observe that at $400 \mathrm{MeV}$ temperature $\gamma_{\mathrm{HTL}}$ charm quark is $\sim 33 \%$ more than the $\gamma$, whereas the corresponding difference is $\sim 25 \%$ for bottom quarks. We also observe that this difference increases with the increase in temperature. Diffusion coefficients, plotted in Figure 23, seem to be more sensitive to the use of effective propagator in a sense that we observe $100 \%$ change between the diffusion using HTL propagator $\left(D_{\mathrm{HTL}}\right)$ and that in $T=0$ pQCD $(D)$ at $T=400 \mathrm{MeV}$ and this difference increases with $T$. Though unlike drag, this difference is not much (3.5\%) for a difference in charm and bottom quark masses.

The authors of [47] also calculate the energy loss of heavy quarks using HTL propagator and get a drag which is $16 \%$ less than that obtained in the present paper at HQ momentum $4 \mathrm{GeV}$ and $T=250 \mathrm{MeV}$. The authors of [18] calculates diffusion coefficient of a nonrelativistic heavy quark in leading order as well as in next to leading order. The leading order result surpasses the present result by $25 \%$ at $T=300 \mathrm{MeV}$ and at a very low momentum $(0.2 \mathrm{GeV})$ of HQ.

However, radiative transport coefficients like drag are also needed for we have seen that radiation becomes very important in high energy regime. We can even extend the present calculation of collisional drag and diffusion using HTL propagator to radiative domains, but that will increase the complexity of problem.

\section{E. Recent Efforts of Generalizing GB Formula}

Gunion-Bertsch formula is derived in the mid-rapidity region and there is another very recent development in this field proposed in [110] where a generalized Gunion-Bertsch formula for arbitrary forward and backward rapidity region has been derived. This modification will be needed when one needs to compute cross sections and rates. Now, this calculation involves two parts (a) keeping a factor $(1-x)^{2}$, where $x$ is the fraction of light cone momentum carried by the emitted gluon and (b) combination of calculations obtained from both $A^{+}=0$ and $A^{-}=0$ gauge conditions for emitted gluon polarization. The final form of $M_{q q^{\prime} \rightarrow q q^{\prime} q}$ can be written as follows:

$$
\left|M_{q q^{\prime} \rightarrow q q^{\prime} g}\right|^{2}=12 g^{2}\left|M_{q q^{\prime} \rightarrow q q^{\prime}}\right|_{s a}^{2}(1-\bar{x})^{2} \times \frac{q_{\perp}^{2}}{k_{\perp}^{2}\left(\vec{q}_{\perp}-\vec{k}_{\perp}\right)^{2}},
$$

where

$$
\bar{x}=\frac{k_{\perp} e^{|\eta|}}{\sqrt{s}}
$$

where $\eta$ is the rapidity of the emitted gluon. With this, the exact differential cross section $(d \sigma / d \eta)$ for the process $q q^{\prime} \rightarrow$ $q q^{\prime} g$ is shown to be reproduced by using (E.1) for all rapidity ranges.

In all these calculations, we tacitly assume that the incoming jet is hard enough so that eikonal approximation (straight path) for it is always valid. But there has been a

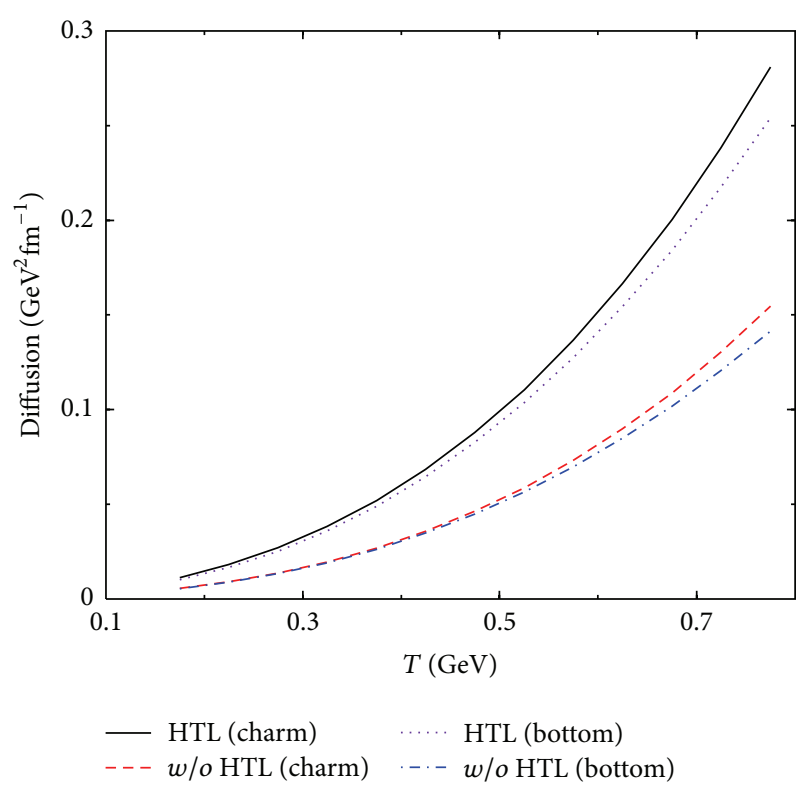

Figure 23: Variation of diffusion of heavy quarks of momentum $1 \mathrm{GeV}$ with temperature (color online).

recent attempt of relaxing the eikonal approximation in [111] and $15 \%-20 \%$ suppression in the differential cross section of $2 \rightarrow 3$ processes for moderately hard jets because of the noneikonal effects that have been found.

\section{F. Dead-Cone Effect Revisited and Other Aspects of Energy Loss}

The authors of [112] compute the HQ-LQ $\rightarrow$ HQ-LQ-g scattering amplitude for soft gluon emission and find out a general expression for radiative suppression factor for deadcone effect. In the limit $m \ll \sqrt{s}$ and $\theta \rightarrow 0$, they reproduce (25). In the backward rapidity region, the gluon emission does not depend on the mass and in $\theta \sim \pm \pi$ region there is no suppression, in contrast with what we get from [71] (see [73] also). The authors of [113] evaluate the HQ energy loss employing the generalized dead-cone factor in [112]. They report similar energy loss for both massless and massive quark jets.

However, the high energy quarks and gluons produced from the hard collisions of the partons from the colliding nucleons are off-shell and their colour fields are stripped off; that is, they have no field to radiate. Therefore, the parton virtuality creates its own dead cone which may be large depending on the magnitude of the virtuality. The forbidden zone around the direction of motion of the partons due to its virtuality will here be called virtual dead cone. If the virtuality does not disappear before the hadronization of the QGP, then the dead cone suppression due to virtuality will play a decisive role in QGP diagnostics by jet quenching. The conventional dead-cone (due to the mass of the quark) becomes important when the virtuality of the quarks reduces to zero.

For the demonstration of suppression of soft gluon radiation due to virtuality, we can take up the $e^{+} e \rightarrow Q \bar{Q} g$ process, 


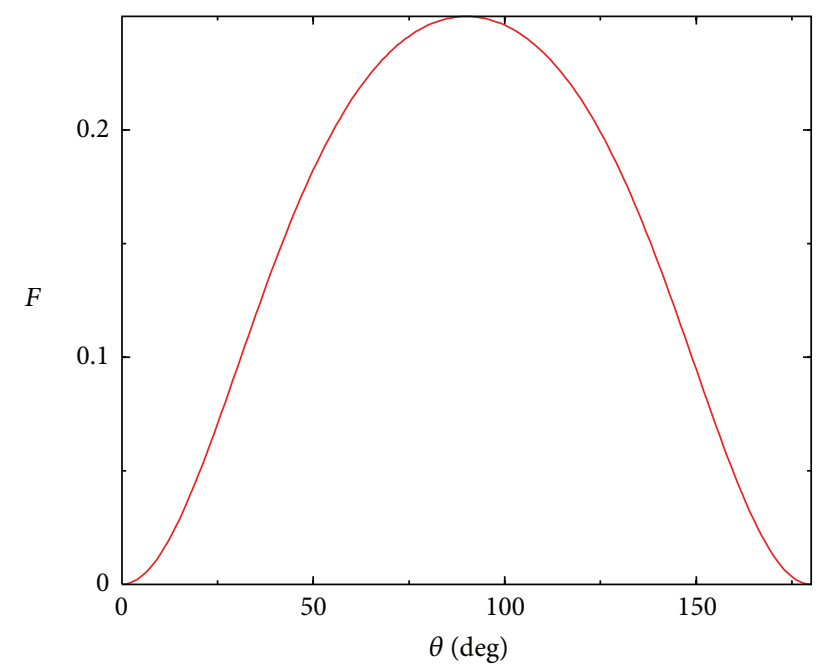

FIgURE 24: Plot of the spectrum $F$ in $V=0$ limit (F.2), $m=1.5 \mathrm{GeV}$, $\beta=0.5$.

where $Q$ is heavy quark. The spectrum of the soft gluons emitted by the virtual quarks can be shown to be [114]

$$
F=4 \beta^{2}\left(\frac{V^{4} / k_{0}^{2} E^{2}+4 V^{2} / k_{0} E+4 \sin ^{2} \theta}{\left(V^{4} / k_{0}^{2} E^{2}+4 V^{2} / k_{0} E+4\left(1-\beta^{2} \cos ^{2} \theta\right)\right)^{2}}\right),
$$

where external quarks are assumed to be on the verge of being on-shell so that Dirac's equation can be applied. $V$ is the virtuality parameter defined by the equation, $V^{2}=q^{2}-$ $m^{2}$ where $q^{2}$ is four-momentum square of external virtual particles and $m$ is its mass, if any. $q^{2}=m^{2}$ implies $V=0$; that is, the particle becomes on-shell. One can show that the spectrum is that of gluons emitted from on-shell quarks when $V=0 . k_{0}$ is the energy of the soft gluon emitted at angle $\theta$ with the parent quark whose velocity is $\beta$ and energy is $E$. The virtuality is replaced by $V=\sqrt{q^{2}-m^{2}}=\sqrt{E^{2}-p^{2}-m^{2}}$. The emitted gluon carry a fraction of parent parton energy consistent with $\left|\vec{p}_{i}\right| \sin \theta \gg k_{0}$ (for dominance of soft gluon emission). Different limits of $F$ given in (F.1) will be worth exploring.

(i) For zero virtuality $(V=0)$ of the massive quark, (F.1) reduces to the conventional dead-cone factor (Figure 24) as

$$
F \rightarrow \frac{\beta^{2} \sin ^{2} \theta}{\left(1-\beta^{2} \cos ^{2} \theta\right)^{2}}
$$

This is the well-known conventional dead cone for a gluon emitted by a massive quark for large angles. The divergence of the factor is shielded by the quark mass or virtuality through $\beta(<1)$. In fact, for on-shell quarks with small $\theta$, one can show that (F.2) (see [71]) boils down to

$$
F=\frac{1}{\theta^{2}} \frac{1}{\left(1+\theta_{0}^{2} / \theta^{2}\right)^{2}}, \quad \theta_{0}=\frac{m}{E}
$$

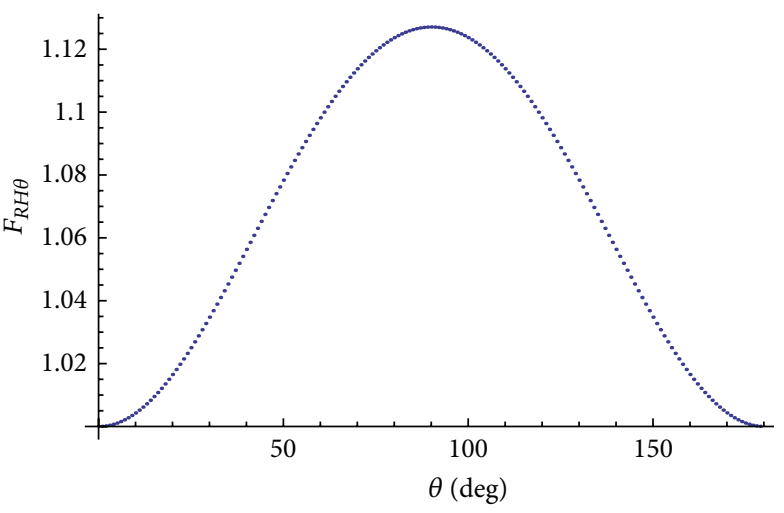

(a)

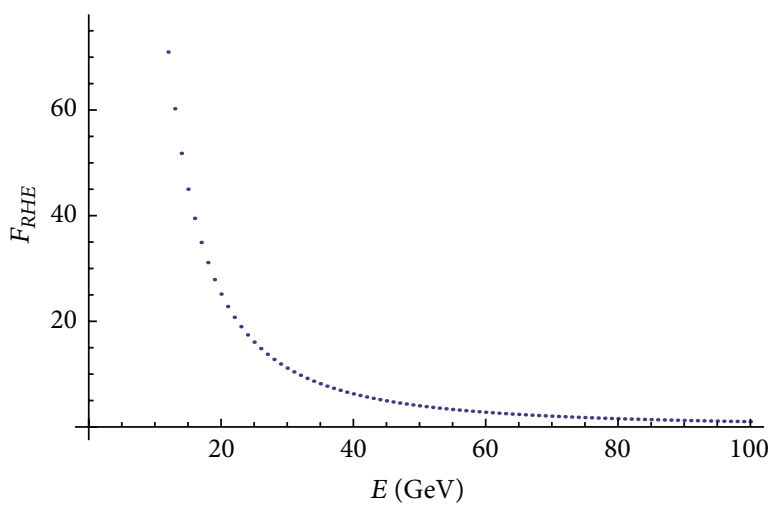

(b)

FIGURE 25: The variation of (a) $F_{R H \theta}(E, \theta)$, defined in the text with $\theta$ for $E=1.5 \mathrm{GeV}$, (b) with $E$ for $\theta=\pi / 4$ for virtual heavy quarks (colour online). The results displayed in this figure are evaluated for heavy quark mass, $m=1.27 \mathrm{GeV}$, and emitted gluon energy, $k_{0}=$ $20 \mathrm{MeV}$ and $\beta=0.5$. The quark virtuality is defined as $V^{2}=E^{2}-$ $\beta^{2} E^{2}-m^{2}$.

and for highly virtual quarks (large $V$ ) the $F$ can be written as:

$$
F=\frac{\omega^{2}}{E^{2}} .
$$

(ii) Now, the light quark limit $(\beta=1)$ can be investigated. For $V=0, \beta=1$,

$$
F \sim \frac{1}{\sin ^{2} \theta} .
$$

For light quarks, (F.5) ensures the absence of deadcone suppression at $\theta=0$ and $\pi$ for vanishing virtuality.

In Figure 25, the suppression of the energy loss, $F$ for heavy quarks is displayed. The variation of $F_{R H \theta}=F(E=$ $1.5 \mathrm{GeV}, \theta) / F(E=1.5 \mathrm{GeV}, \theta=0)$ with $\theta$ is depicted in Figure 26(a). It is interesting to note that for vanishingly small virtuality the suppression is similar to that of a conventional dead cone that appears for massive on-shell quarks (Figure 24). In Figure 25(b), the variation of $F_{R H E}=F(E, \theta=$ $\pi / 4) / F(E=100 \mathrm{GeV}, \theta=\pi / 4)$ with $E$ is displayed for heavy 
quarks. For large virtuality (which increases with parton energy, $E$ ), the suppression is large.

Figure 26 illustrates the suppression of the energy loss for light quarks. In Figure 26(a), the variation of $F_{R L \theta}=F(E=$ $3 \mathrm{GeV}, \theta) / F(E=3 \mathrm{GeV}, \theta=0)$ with $\theta$ is shown for light partons. It is important to note that the variation of $F_{R L \theta}$ with $\theta$ for light quark with low virtuality is drastically different from the corresponding quantity, $F_{R H \theta}$, for heavy quark. This is obvious because for low virtuality the light partons are not subjected to any dead-cone suppression at $\theta=0$ and $\pi$ unlike heavy quarks. Moreover, the $\sin ^{-2} \theta$ behaviour for light quarks (F.5) ensures a minimum at $\theta=\pi / 2$ as opposed to a maximum at the same $\theta$ for heavy quarks. In Figure 26(b), the variation of $F_{R L E}=F(E, \theta=\pi / 4) / F(E=100 \mathrm{GeV}, \theta=\pi / 4)$ with $E$ is depicted. We note that the suppression is large for high $E$ and the behaviour of $F_{R L E}$ is similar to $F_{R H E}$ which indicates that the suppression due to virtuality overwhelm the effects due to the conventional dead-cone.

The energy loss $(\Delta E(L))$ of quarks as a function of path length $(L)$ traversed by the off-shell parton in vacuum can be evaluated (see [115] for details) by using the emitted gluon spectrum given by (F.1). The results are displayed in Figure 27. We note that the energy loss of light and heavy quarks differ significantly at large path length or time when the propagating quarks acquire enough field to radiate. However, at small path length, the value of $\Delta E$ for light and heavy quarks is similar.

When we want to talk about radiative energy loss of high energy partons, we mean that the absorption of radiation given off is absorbed in the medium. So, we must take into account the interaction of emitted radiation with the medium. Consequently, the dispersion relation of the emitted gluon should change. This change in gluon dispersion relation is encoded in the thermal quark self-energy, and as the inverse of imaginary part of self energy gives radiation production rate, the formation time of radiation is also modified. This effect of modified dispersion relation of emitted gluon (or photon) is called Ter-Mikaelian (TM) effect [69]. The QCD analogue of TM effect is discussed in [116, 117]. The authors of [56] implement TM effect by replacing $x^{2} m^{2}$ by $x^{2} m^{2}+$ $(1-x) m_{D}^{2}$, where $m_{D}$ is thermal mass of gluon. However, the authors of [116] repeats the single, double, and multiple scattering calculations of [49] by assuming a modified dispersion relation, $k^{2}=\omega_{0}^{2}(T)$, of emitted gluon. $\omega_{0}$ parametrizes gluon self-energy in medium. The authors of [116] show that in the phase space region where abelian radiation does not occur, the gluon radiation is suppressed due to polarization properties of the medium. The authors of $[117,118]$ extend the results of [116] using 1-Loop HTL self-energy of gluons. The authors of [118] show a 30\% decrease in the 1st order in opacity fractional energy loss for heavy quarks of $10 \mathrm{GeV}$ energy when TM effect is taken into account, whereas the authors of [56] show $\sim 1.5$ times increase in radiative energy spectra per unit length when gluon thermal mass is taken into consideration. Such changes indicate that one can barely neglect the effect of gluon interaction with medium while probing QGP.

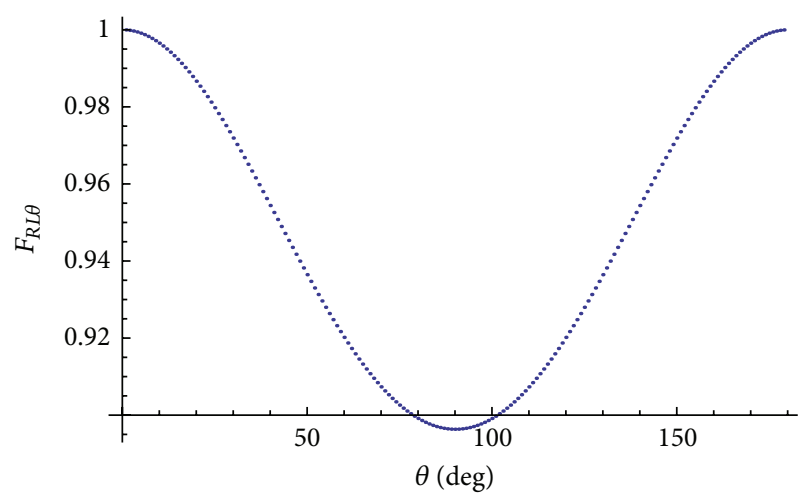

(a)

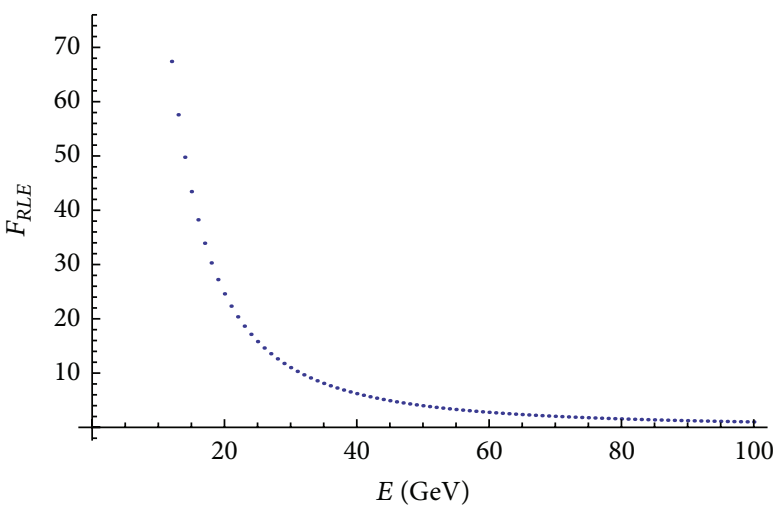

(b)

FIgURE 26: The variation of (a) $F_{R L \theta}(E, \theta)$, defined in the text with $\theta$ for $E=3 \mathrm{GeV}$, (b) with $E$ for $\theta=\pi / 4$ for virtual light partons (colour online). The results displayed in this figure are evaluated for vanishing quark mass, $\beta=0.95$, and emitted gluon energy, $k_{0}=$ $10 \mathrm{MeV}$. The quark virtuality is defined as $V^{2}=E^{2}\left(1-\beta^{2}\right)$.

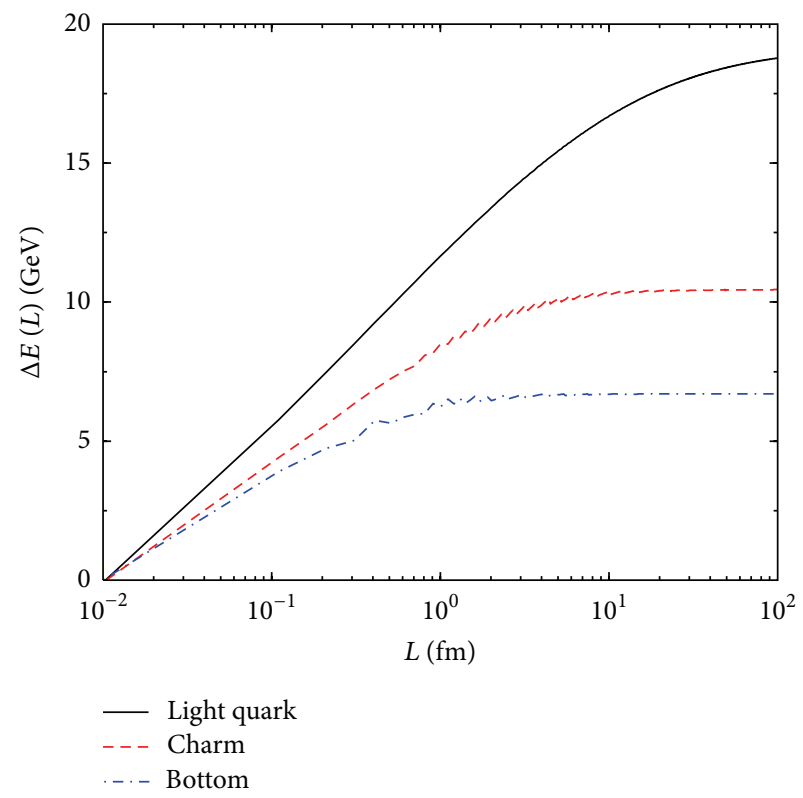

Figure 27: Variation of energy loss, $\Delta E$ as a function of path traversed, $L$ in vacuum. The energy of the quark is taken as $E=$ $20 \mathrm{GeV}$. 
So far, we have talked about the energy loss of HQs in an infinite medium. But, how does the energy loss depend on the size of medium? The authors show that length dependent energy loss $\Delta E(L)$ is $\propto L^{2}$, when we are considering the coherent region; that is, the emitted gluon energy is soft and is within the factorization and the Bethe-Heitler limits (see [119]). When gluon energy $\left(k_{0}\right)$ is of the order of that of parent parton $(E)$, the energy loss per unit length becomes independent of length of medium. This is the limit $\left(k_{0} \sim E\right)$ when evaluation of transport coefficients without addressing to size of medium works more efficiently.

There is also a path integral approach of radiative energy loss proposed in $[120,121]$. Path integral approach is shown to be equivalent to the approach of $[51,52]$ in [119]. An alternate formalism (GLV) proposed in $[53,122]$ performs a systematic expansion in opacity (the mean number of jet scatterings). Opacity is quantitatively given by $\bar{n}=L / \lambda$, where $L$ is the target thickness and $\lambda$ is the mean free path. One analytic limit applies to plasmas where mean number of scattering is small [50]. The other limit applies to thick plasma where $\bar{n} \gg 1[51,52]$.

\section{Acknowledgments}

Surasree Mazumder and Trambak Bhattacharyya are supported by DAE, Government of India. Santosh K. Das acknowledges the support by the ERC StG under the QGPDyn Grant no. 259684. The authors thank Jan-e Alam and Md. Younus for fruitful discussions and critical reading of the paper. The authors also take the opportunity to thank the anonymous reviewers for their relevant suggestions which have undoubtedly helped to increase the quality of paper.

\section{References}

[1] D. J. Gross and F. Wilczek, "Ultraviolet behavior of non-Abelian gauge theories," Physical Review Letters, vol. 30, p. 1343, 1973.

[2] D. J. Gross and F. Wilczek, "Asymptotically free gauge theories. I," Physical Review D, vol. 8, no. 10, pp. 3633-3652, 1973.

[3] H. D. Politzer, "Reliable perturbative results for strong interactions?” Physical Review Letters, vol. 30, p. 1346, 1973.

[4] J. C. Collins and M. J. Perry, "Superdense matter: neutrons or asymptotically free quarks?” Physical Review Letters, vol. 34, p. 1353, 1975.

[5] N. Cabibbo and G. Parisi, "Exponential hadronic spectrum and quark liberation," Physics Letters B, vol. 59, p. 67, 1975.

[6] F. Karsch, "Lattice QCD at high temperature and density," Lecture Notes in Physics, vol. 583, pp. 209-249, 2002.

[7] F. Karsch, "Lattice results on QCD thermodynamics," Nuclear Physics A, vol. 698, pp. 199-208, 2002.

[8] S. Borsányi, G. Endrődi, Z. Fodor et al., “The QCD equation of state with dynamical quarks," Journal of High Energy Physics, vol. 11, p. 077, 2010.

[9] Y. Aoki, G. Endrődi, Z. Fodor, S. D. Katz, and K. K. Szabó, “The order of the quantum chromodynamics transition predicted by the standard model of particle physics," Nature, vol. 443, no. 7112, pp. 675-678, 2006.

[10] S. Borsányi, G. Endrődi, Z. Fodor et al., “Transition temperature and the equation of state from lattice QCD, Wuppertal-Buda- pest results," Journal of Physics G, vol. 38, no. 12, Article ID 124101, 2011.

[11] P. Kovtun, D. T. Son, and O. A. Starinets, "Viscosity in strongly interacting quantum field theories from black hole physics," Physical Review Letters, vol. 94, Article ID 111601, 2005.

[12] A. Adams, L. D. Carr, T. Schaefer, P. Steinberg, and J. E. Thomas, "Strongly correlated quantum fluids: ultracold quantum gases, quantum chromodynamic plasmas, and holographic duality," New Journal of Physics, vol. 14, Article ID 115009, 2012.

[13] S. S. Adler, S. Afanasiev, C. Aidala et al., "Nuclear modification of electron spectra and implications for heavy quark energy loss in $\mathrm{Au}+\mathrm{Au}$ collisions at $\sqrt{S_{N N}}=200 \mathrm{GeV}$," Physical Review Letters, vol. 96, Article ID 032301, 2006.

[14] B. I. Abelev, M. M. Aggarwal, Z. Ahammed et al., “Transverse momentum and centrality dependence of high- $p_{T}$ nonphotonic electron suppression in $\mathrm{Au}+\mathrm{Au}$ collisions at $\sqrt{S_{N N}}=200 \mathrm{GeV}$," Physical Review Letters, vol. 98, Article ID 192301, 2007.

[15] B. Abelev, J. Adam, D. Adamova et al., "Production of muons from heavy flavour decays at forward rapidity in $\mathrm{pp}$ and $\mathrm{Pb}-\mathrm{Pb}$ collisions at $\sqrt{S_{N N}}=2.76 \mathrm{TeV}$,' Physical Review Letters, vol. 109, Article ID 112301, 2012.

[16] A. Rossi, "D meson nuclear modification factors in $\mathrm{Pb}-\mathrm{Pb}$ collisions at $\sqrt{S_{N N}}=2.76 \mathrm{TeV}$, measured with the ALICE detector," in Proceedings of Quark Matter Conference, Annecy, France, May 2011.

[17] A. Adare, S. Afanasiev, C. Aidala et al., "Energy loss and flow of heavy quarks in $\mathrm{Au}+\mathrm{Au}$ collisions at $\sqrt{S_{N N}}=200 \mathrm{GeV}$," Physical Review Letters, vol. 98, Article ID 172301, 2007.

[18] G. D. Moore and D. Teaney, "How much do heavy quarks thermalize in a heavy ion collision?" Physical Review C, vol. 71, Article ID 064904, 2005.

[19] C. M. Ko and W. Liu, "Suppression of heavy quarks in heavy-ion collisions," Nuclear Physics A, vol. 783, pp. 233-240, 2007.

[20] A. Adil and I. Vitev, "Collisional dissociation of heavy mesons in dense QCD matter," Physics Letters B, vol. 649, p. 139, 2007.

[21] N. Armesto, M. Cacciari, A. Dainese, C. A. Salgado, and U. A. Wiedemann, "How sensitive are high- $p_{T}$ electron spectra at RHIC to heavy quark energy loss?" Physics Letters B, vol. 637, p. $362,2006$.

[22] P. B. Gossiaux and J. Aichelin, "Toward an understanding of the single electron data measured at the BNL Relativistic Heavy Ion Collider (RHIC)," Physical Review C, vol. 78, Article ID 014904, 2008.

[23] S. K. Das, J. Alam, and P. Mohanty, "Probing quark gluon plasma properties by heavy flavors," Physical Review C, vol. 80, Article ID 054916, 8 pages, 2009.

[24] S. K. Das, J. Alam, and P. Mohanty, "Drag of heavy quarks in quark gluon plasma at energies available at the CERN Large Hadron Collider (LHC)," Physical Review C, vol. 82, Article ID 014908, 2010.

[25] S. K. Das, J. Alam, P. Mohanty, and B. Sinha, "Nuclear suppression at low energy in relativistic heavy ion collisions," Physical Review C, vol. 81, Article ID 044912, 2010.

[26] J. Uphoff, O. Fochler, Z. Xu, and C. Greiner, "Elliptic flow and energy loss of heavy quarks in ultrarelativistic heavy ion collisions," Physical Review C, vol. 84, Article ID 024908, 2011.

[27] W. M. Alberico, A. Beraudo, A. De Pace et al., "Heavy-flavour spectra in high-energy nucleus-nucleus collisions," The European Physical Journal C, vol. 71, p. 1666, 2011.

[28] S. Mazumder, T. Bhattacharyya, J. Alam, and S. K. Das, "Momentum dependence of drag coefficients and heavy flavor 
suppression in quark gluon plasma," Physical Review C, vol. 84, Article ID 044901, 2011.

[29] M. Younus and D. K. Srivastava, "Empirical determination of charm quark energy loss and its consequences for azimuthal anisotropy," Journal of Physics G, vol. 39, Article ID 095003, 2012.

[30] M. G. Mustafa, D. Pal, D. K. Srivastava, and M. H. Thoma, "Radiative energy-loss of heavy quarks in a quark-gluon plasma," Physics Letters B, vol. 428, p. 234, 1998.

[31] C. Young, B. Schenke, S. Jeon, and C. Gale, "MARTINI event generator for heavy quarks: Initialization, parton evolution, and hadronization," Physical Review C, vol. 86, Article ID 034905, 7 pages, 2012.

[32] S. Cao, G. Qin, and S. Bass, "A systematic study of heavy quark energy loss in a hot and dense QCD medium," http://arxiv.org/ abs/1205.2396.

[33] T. Lang, H. Hees, J. Steinheimer, and M. Bleicher, "Charm quark transport in $\mathrm{Pb}+\mathrm{Pb}$ reactions at $\sqrt{S_{N N}}=2.76 \mathrm{TeV}$ from a $(3+1)$ dimensional hybrid approach," http://arxiv.org/abs/1208.1643.

[34] S. K. Das, V. Chandra, and J. Alam, "Heavy-quark transport coefficients in a hot viscous quark-gluon plasma medium," http:// arxiv.org/abs/1210.3905.

[35] S. Chakravorty and D. Syam, "Pre-thermalization quark momentum distribution in the quark-gluon plasma," Lettere Al Nuovo Cimento Series 2, vol. 41, no. 11, p. 381, 1984.

[36] J. Alam, B. Sinha, and S. Raha, "Successive equilibration in quark-gluon plasma," Physical Review Letters, vol. 73, p. 1895, 1994.

[37] B. Svetitsky, "Diffusion of charmed quarks in the quark-gluon plasma," Physical Review D, vol. 37, no. 9, pp. 2484-2491, 1988.

[38] H. M. Antia, Numerical Methods for Scientists and Engineers, Tata McGraw-Hill, 1991.

[39] M. L. Mangano, P. Nason, and G. Ridolfi, "Heavy-quark correlations in hadron collisions at next-to-leading order," Nuclear Physics B, vol. 373, no. 2, pp. 295-345, 1992.

[40] H. van Hees and R. Rapp, "Thermalization of heavy quarks in the quark-gluon plasma," Physical Review C, vol. 71, Article ID 034907, 2005.

[41] B. L. Combridge, "Associated production of heavy flavour states in pp and $\bar{p}$ p interactions: some QCD estimates," Nuclear Physics B, vol. 151, p. 429, 1979.

[42] M. G. Mustafa, D. Pal, and D. K. Srivastava, "Propagation of charm quarks in equilibrating quark-gluon plasma," Physical Review C, vol. 57, p. 889, 1998.

[43] H. Van Hees, M. Mannarelli, V. Greco, and R. Rapp, “T-matrix approach to heavy quark diffusion in the QGP," European Physical Journal C, vol. 61, no. 4, pp. 799-806, 2009.

[44] P. B. Gossiaux, J. Aichelin, and T. Gousset, "Theory of heavy quark energy loss," Progress of Theoretical Physics Supplements, vol. 193, p. 110, 2012.

[45] M. He, R. J. Fries, and R. Rapp, "Heavy-quark diffusion and hadronization in quark-gluon plasma," Physical Review C, vol. 86, Article ID 014903, 15 pages, 2012.

[46] W. R. Leo, Techniques for Nuclear and Particle Physics Experiments, Springer, Berlin, Germany, 1987.

[47] E. Braaten and M. H. Thoma, "Energy loss of a heavy quark in the quark-gluon plasma," Physical Review D, vol. 44, p. R2625, 1991.

[48] M. Gyulassy, M. Plumer, M. H. Thoma, and X. N. Wang, "High $p_{T}$ probes of nuclear collisions," Nuclear Physics A, vol. 538, p. 37c, 1992.
[49] X. N. Wang, M. Gyulassy, and M. Plümer, "Landau-Pomeranchuk-Migdal effect in QCD and radiative energy loss in a quark-gluon plasma," Physical Review D, vol. 51, p. 3436, 1995.

[50] M. Gyulassy and X.-N. Wang, "Multiple collisions and induced gluon bremsstrahlung in QCD," Nuclear Physics B, vol. 420, no. 3, pp. 583-614, 1994.

[51] R. Baier, Yu. L. Dokshitzer, S. Peigné, and D. Schiff, "Induced gluon radiation in a QCD medium," Physics Letters B, vol. 345, p. 277, 1995.

[52] R. Baier, Yu. L. Dokshitzer, A. H. Mueller, S. Peigné, and D. Schiff, "Radiative energy loss of high energy quarks and gluons in a finite-volume quark-gluon plasma," Nuclear Physics B, vol. 483, pp. 291-320, 1997.

[53] M. Gyulassy, P. Levai, and I. Vitev, "Non-abelian energy loss at finite opacity," Physical Review Letters, vol. 85, no. 26, pp. 5535$5538,2000$.

[54] L. D. Landau and I. Ya. Pomeranchuk, "Electron-avalanche process at high energies," Doklady Akademii Nauk SSSR, vol. 92, p. $535,735,1953$.

[55] A. B. Migdal, "Bremsstrahlung and pair production in condensed media at high energies," Physical Review, vol. 103, p. 1811, 1956.

[56] P. B. Gossiaux, J. Aichelin, T. Gousset, and V. Guiho, "Competition of heavy quark radiative and collisional energy loss in deconfined matter," Journal of Physics G: Nuclear and Particle Physics, vol. 37, no. 9, Article ID 094019, 2010.

[57] Y. S. Kim and M. Noz, "Dirac's light-cone coordinate system," American Journal of Physics, vol. 50, no. 8, pp. 721-724, 1982.

[58] L. McLerran, "Three lectures on the physics of small $\mathrm{x}$ and high gluon density," in Proceedings of the 8th Mexican School on Particles and Fields, vol. 490 of AIP Conference Proceedings, pp. 42-79, 1998.

[59] R. Thomas, Energy loss of quarks by gluon radiation in deconfined matter [Ph.D. thesis], Institut für Theoretische Physik, 2003.

[60] J. F. Gunion and G. Bertsch, "Hadronization by color bremsstrahlung," Physical Review D, vol. 25, no. 3, pp. 746-753, 1982.

[61] S. K. Das and J. Alam, "Soft gluon multiplicity distribution revisited," Physical Review D, vol. 82, Article ID 051502(R), 2010.

[62] R. Abir, C. Greiner, M. Martinez, and M. G. Mustafa, "Generalization of Gunion-Bertsch formula for soft gluon emission," Physical Review D, vol. 83, Article ID 011501, 2011.

[63] T. Bhattacharyya, S. Mazumder, S. K. Das, and J. Alam, "Examination of the Gunion-Bertsch formula for soft gluon radiation," Physical Review D, vol. 85, Article ID 034033, 2012.

[64] F. A. Berends, R. Kleiss, P. De Causmaecker, R. Gastmans, and T. T. Wu, "Single bremsstrahlung processes in gauge theories," Physics Letters B, vol. 103, p. 124, 1981.

[65] S. M. H. Wong, "Thermal and chemical equilibration in a gluon plasma," Nuclear Physics A, vol. 607, p. 442, 1996.

[66] M. Le Bellac, Thermal Field Theory, Cambridge Monographs on Mathematical Physics, Cambridge University Press, Cambridge, UK, 1996.

[67] J. I. Kapusta and C. Gale, Finite-Temperature Field Theory, Cambridge Monographs on Mathematical Physics, Cambridge University Press, Cambridge, UK, 2nd edition, 2006.

[68] O. Kaczmarek and F. Zantow, "Static quark-antiquark interactions in zero and finite temperature QCD: I. Heavy quark free energies, running coupling, and quarkonium binding," Physical Review D, vol. 71, Article ID 114510, 2005. 
[69] S. Klein, "Suppression of bremsstrahlung and pair production due to environmental factors," Reviews of Modern Physics, vol. 71, p. 1501, 1999.

[70] M. H. Thoma, "Parton interaction rates in the quark-gluon plasma," Physical Review D, vol. 49, p. 451, 1994.

[71] Y. L. Dokshitzer and D. E. Kharzeev, "Heavy-quark colorimetry of QCD matter," Physics Letters, Section B: Nuclear, Elementary Particle and High-Energy Physics, vol. 519, no. 3-4, pp. 199-206, 2001.

[72] J. D. Jackson, Classical Electrdynamics, John Wiley \& Sons, New York, NY, USA, 1998.

[73] K. M. Paul, From Higgs to top to color [Ph.D. thesis], University of Illinois at Urbana-Champaign, 2002.

[74] J. D. Bjorken, "Highly relativistic nucleus-nucleus collisions: the central rapidity region," Physical Review D, vol. 27, p. 140, 1983.

[75] S. Turbide, C. Gale, S. Jeon, and G. D. Moore, "Energy loss of leading hadrons and direct photon production in evolving quark-gluon plasma," Physical Review C, vol. 72, Article ID 014906, 2005.

[76] C. Peterson, D. Schlatter, I. Schmitt, and P. M. Zerwas, "Scaling violations in inclusive $e^{+} e^{-}$annihilation spectra," Physical Review D, vol. 27, p. 105, 1983.

[77] R. J. Fries, V. Greco, and P. Sorensen, "Coalescence models for hadron formation from quark-gluon plasma," Annual Review of Nuclear and Particle Science, vol. 58, p. 177, 2008.

[78] V. Greco, H. van Hees, and R. Rapp, "Heavy quark dynamics in the QGP," in International Workshop on Early Physics with Heavy-Ion Collisions at the LHC, vol. 1422 of AIP Conference Proceedings, pp. 117-126, 2012.

[79] R. C. Hwa and K. Kajantie, "Diagnosing quark matter by measuring the total entropy and the photon or dilepton emission rates," Physical Review D, vol. 32, p. 1109, 1985.

[80] S. Mazumder and J. Alam, "Effects of equation of state on nuclear suppression and the initial entropy density of quark gluon plasma," Physical Review C, vol. 85, Article ID 044918, 2012.

[81] T. Sakaguchi, "PHENIX plans for RHIC low energy run," in Proceedings of the 5th International Workshop on Critical Point and Onset of Deconfinement (CPOD '09), Long Island, NY, USA, June 2009.

[82] B. I. Abelev, "Identified particle production, azimuthal anisotropy, and interferometry measurements in $\mathrm{Au}+\mathrm{Au}$ collisions at $\sqrt{S_{N N}}=9.2 \mathrm{GeV}$," Physical Review $C$, vol. 81, Article ID 024911, 2010.

[83] J. M. Heuser, "The compressed baryonic matter experiment at FAIR: physics of strangeness and charm, status of preparations," Journal of Physics G, vol. 35, Article ID 044049, 2008.

[84] O. Ristea, "Study of the chemical freeze-out in nucleus-nucleus collisions," Romanian Reports in Physics, vol. 56, pp. 659-666, 2004.

[85] A. Andronic, P. Braun-Munzinger, and J. Stachel, "Hadron production in central nucleus-nucleus collisions at chemical freeze-out," Nuclear Physics A, vol. 772, no. 3-4, pp. 167-199, 2006.

[86] D. Kharzeev and M. Nardi, "Hadron production in nuclear collisions at RHIC and high-density QCD," Physics Letters B, vol. 507, p. 121, 2001.

[87] R. J. Glauber, "High-energy collision theory," in Lectures in Theoretical Physics, Vol. I. Lectures Delivered at the Summer Institute for Theoretical Physics, University of Colorado, Boulder, 1958, W. E. Brittin and L. G. Dunham, Eds., pp. 315-414, Interscience Publishers, New York, NY, USA, 1959.
[88] B. B. Back, M. D. Baker, M. Ballintijn et al., "Collision geometry scaling of $\mathrm{Au}+\mathrm{Au}$ pseudorapidity density from $\sqrt{S_{N N}}=19.6$ to 200 GeV," Physical Review C, vol. 70, Article ID 021902, 2004.

[89] R. D. Field, Application of Perturbative QCD, Addison-Wesley, New York, NY, USA, 1989.

[90] T. Hirano, N. van der Kolk, and A. Bilandzic, "Hydrodynamics and flow," Lecture Notes in Physics, vol. 785, pp. 139-178, 2010.

[91] P. Huovinen and P. V. Ruuskanen, "Hydrodynamic models for heavy ion collisions," Annual Review of Nuclear and Particle Science, vol. 56, p. 163, 2006.

[92] D. A. Teaney, "Viscous hydrodynamics and the quark gluon plasma," http://arxiv.org/abs/0905.2433.

[93] H. van Hees, V. Greco, and R. Rapp, "Heavy-quark probes of the quark-gluon plasma and interpretation of recent data taken at the BNL Relativistic Heavy Ion Collider," Physical Review C, vol. 73, Article ID 034913, 2006.

[94] H. van Hees, M. Mannarelli, V. Greco, and R. Rapp, "Nonperturbative heavy-quark diffusion in the quark-gluon plasma," Physical Review Letters, vol. 100, no. 19, Article ID 192301, 2008.

[95] Y. Akamatsu, T. Hatsuda, and T. Hirano, "Heavy quark diffusion with relativistic Langevin dynamics in the quark-gluon fluid," Physical Review C, vol. 79, Article ID 054907, 2009.

[96] S. K. Das and J. Alam, "Elliptic flow of heavy flavors," http:// arxiv.org/abs/1008.2643.

[97] S. K. Das and A. Davody, "A non-conformal holographic model for D-meson suppression at LHC energy," http://arxiv.org/abs/ 1211.2925.

[98] S. S. Gubser, "Drag force in AdS/CFT”, Physical Review D, vol. 74, no. 12, Article ID 126005, 4 pages, 2006.

[99] G. C. Giecold, "Heavy quark in an expanding plasma in AdS/ CFT," Journal of High Energy Physics, vol. 06, p. 002, 2009.

[100] M. G. Mustafa, "Energy loss of charm quarks in the quark-gluon plasma: Collisional vs radiative losses," Physical Review C, vol. 72, Article ID 014905, 2005.

[101] M. Laine, "Heavy flavour kinetic equilibration in the confined phase," Journal of High Energy Physics, vol. 04, p. 124, 2011.

[102] M. He, R. J. Fries, and R. Rapp, "Thermal relaxation of charm in hadronic matter," Physics Letters B, vol. 701, p. 445, 2002.

[103] S. Ghosh, S. K. Das, S. Sarkar, and J. Alam, "Drag coefficient of $D$ mesons in hot hadronic matter," Physical Review D, vol. 84, Article ID 011503, 2011.

[104] L. M. Abreu, D. Cabrera, F. J. Llanes-Estrada, and J. M. TorresRincon, "Charm diffusion in a pion gas implementing unitarity, chiral and heavy quark symmetries," Annals of Physics, vol. 326, no. 10, pp. 2737-2772, 2011.

[105] L. Abreu, D. Cabrera, and J. M. Torres-Rincon, "Transport properties of bottomed mesons in a hot mesonic gas," Physical Review D, vol. 87, Article ID 034019, 2013.

[106] S. K. Das, S. Ghosh, S. Sarkar, and J. Alam, "Drag and diffusion coefficients of B mesons in hot hadronic matter," Physical Review D, vol. 85, Article ID 074017, 2012.

[107] D. Cabrera, L. M. Abreu, F. J. Llanes-Estrada, and J. M. TorresRincon, "Heavy flavor relaxation in a hadronic medium," $\mathrm{Nu}$ clear Physics A, 2013.

[108] S. K. Das, S. Ghosh, S. Sarkar, and J. Alam, "Heavy flavor suppression: role of hadronic matter," http://arxiv.org/abs/1303. 2476.

[109] H. Heiselberg and X.-N. Wang, "Thermal equilibration in an expanding parton plasma," Nuclear Physics B, vol. 462, no. 2-3, pp. 389-411, 1996. 
[110] O. Fochler, J. Uphoff, Z. Xu, and C. Greiner, "Radiative parton processes in perturbative QCD-an improved version of the Gunion and Bertsch cross section from comparisons to the exact result," http://arxiv.org/abs/1302.5250.

[111] R. Abir, "Jet-parton inelastic interaction beyond eikonal approximation,” Physical Review D, vol. 87, Article ID 034036, 2013.

[112] R. Abir, C. Greiner, M. Martinez, M. G. Mustafa, and J. Uphoff, "Soft gluon emission off a heavy quark revisited," Physical Review D, vol. 85, no. 5, Article ID 054012, 2012.

[113] R. Abir, U. Jamil, M. G. Mustafa, and D. K. Srivastava, "Heavy quark energy loss and D-mesons in RHIC and LHC energies," Physics Letters B, vol. 715, p. 183, 2012.

[114] T. Bhattacharyya and J. Alam, "Dead cone due to parton virtuality," International Journal of Modern Physics A, vol. 28, Article ID 1350031, 2013.

[115] B. Z. Kopeliovich, I. K. Potashnikova, and I. Schmidt, "Why heavy and light quarks radiate energy with similar rates," Physical Review C, vol. 82, Article ID 037901, 2010.

[116] B. Kampfer and O. Pavlenko, "The Ter-Mikaelian and LandauPomeranchuk effects for induced soft gluon radiation in a QCD medium," Physics Letters B, vol. 477, p. 171, 2000.

[117] M. Djordjevic and M. Gyulassy, "Where is the charm quark energy loss at RHIC?" Physics Letters B, vol. 560, pp. 37-43, 2003.

[118] M. Djordjevic, "Ter-Mikayelian effect on QCD radiative energy loss," Physical Review C, vol. 68, Article ID 034914, 2003.

[119] R. Baier, D. Schiff, and B. G. Zakharov, "Energy loss in perturbative QCD," Annual Review of Nuclear and Particle Science, vol. 50, p. 37, 2000.

[120] B. G. Zakharov, "Fully quantum treatment of the LandauPomeranchik-Migdal effect in QED and QCD," Journal of Experimental and Theoretical Physics Letters, vol. 63, pp. 952-957, 1996.

[121] B. G. Zakharov, "Landau-Pomeranchuk-Migdal effect for finitesize targets," Journal of Experimental and Theoretical Physics Letters, vol. 64, p. 781, 1996.

[122] M. Gyulassy, P. Levai, and I. Vitev, "Reaction operator approach to non-abelian energy loss," Nuclear Physics B, vol. 594, no. 1-2, pp. 371-419, 2001. 

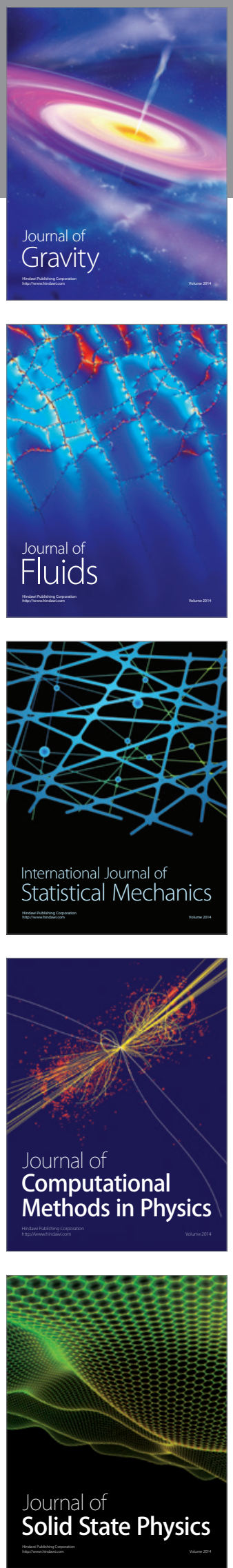

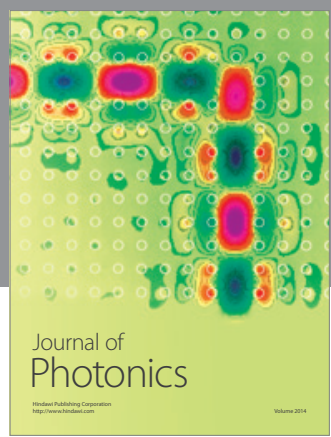

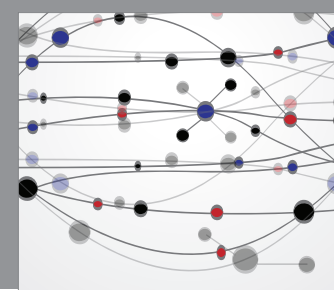

The Scientific World Journal

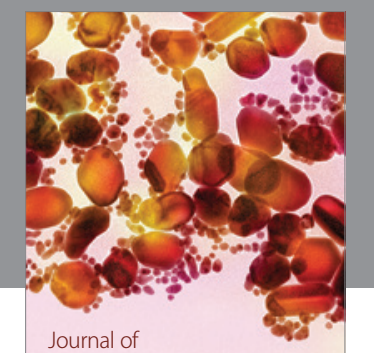

Soft Matter
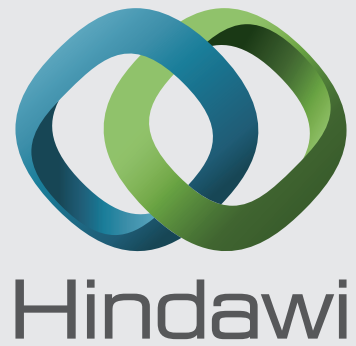

Submit your manuscripts at

http://www.hindawi.com
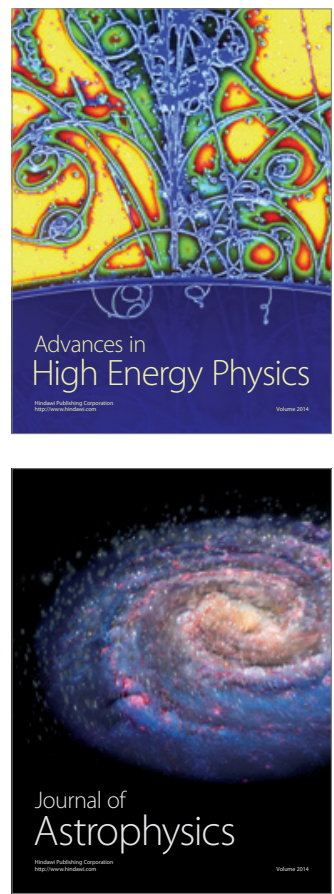
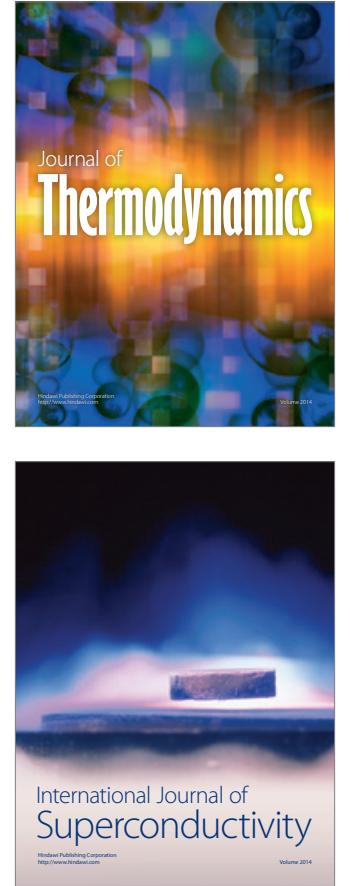
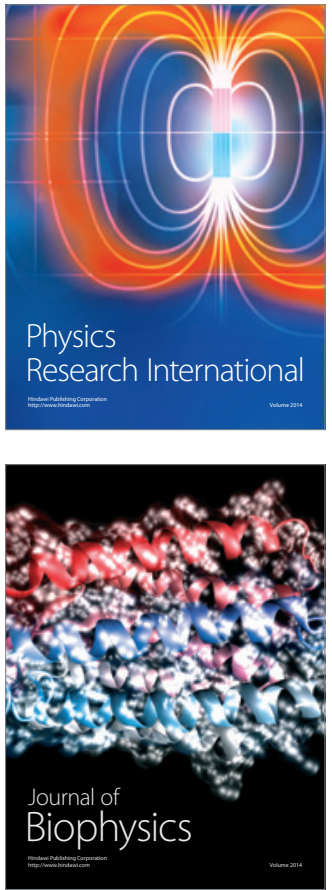
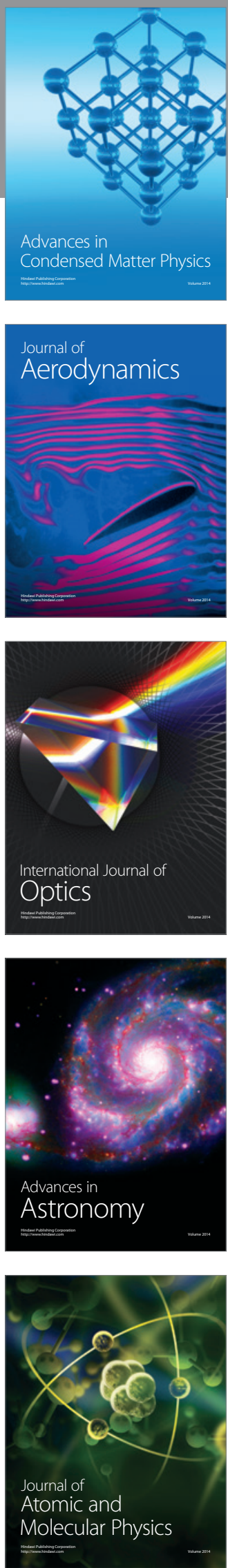